\title{
Acute Inactivation of Tau Has No Effect on Dynamics of Microtubules in Growing Axons of Cultured Sympathetic Neurons
}

\author{
Irina Tint, ${ }^{1}$ Theresa Slaughter, ${ }^{1}$ Itzhak Fischer, ${ }^{2}$ and Mark M. Black ${ }^{1}$ \\ 1 Department of Anatomy and Cell Biology, Temple University School of Medicine, Philadelphia, Pennsylvania 19140, and \\ 2Department of Neurobiology and Anatomy, Allegheny University of the Health Sciences, Philadelphia, Pennsylvania 19129
}

\begin{abstract}
Tau is a developmentally regulated microtubule (MT)associated protein in neurons that has been implicated in neuronal morphogenesis. On the basis of test tube studies, tau has been proposed to function in axon growth by stabilizing MTs and thereby promoting MT assembly. We have tested this hypothesis by examining the effects of acute inactivation of tau on axonal MTs. Tau was inactivated by microinjecting purified antibodies against recombinant tau into neurons before they extended axons. The injected antibodies quantitatively precipitated tau into aggregates in the soma. With these conditions the neurons elaborate normal-appearing axons, and MTs extend throughout the axons and into the growth cones, but the axons and their MTs are depleted of tau. The immunodepletion of tau had no detectable effect on several parameters of the
\end{abstract}

dynamics of axonal MTs. Depletion of tau also was not accompanied by a reorganization of other major MT-associated proteins or actin filaments in these neurons. Thus, neurons effectively depleted of tau can extend axons that resemble those of control cells, and the axons contain normal-appearing MT arrays with normal dynamic behavior. These observations are exactly the opposite of those expected on the basis of the hypothesis that the stability of axonal MTs is a direct function of their content of tau, indicating that tau in growing axons of cultured sympathetic neurons is not specialized to promote microtubule assembly and stability.

Key words: tau; microtubule-associated proteins; microtubule dynamics; axon growth; microinjection; quantitative digital image analysis; cultured sympathetic neurons
Specialized microtubule (MT) arrays are generated by growing neurons that are essential to axonal morphogenesis. Most, if not all, axonal MTs initially are assembled in the neuron soma, and then they are actively transported into the axon by specific motor proteins (Baas and Brown, 1997; Slaughter et al., 1997). This MT transport provides a steady supply of new MTs for the growing axon and also establishes the plus-end distal polarity orientation of axonal MTs by conveying MTs specifically with their plus ends leading (Baas and Brown, 1997). Most or all transported MTs in growing axons are also dynamically active at their plus ends, gaining and losing subunits while in transit toward the axon tip (Slaughter et al., 1997). These local dynamics combine with MT transport to establish the architecture of the axonal MT array (Black, 1994).

The mechanisms that regulate MT dynamics within growing axons are unknown. In this regard, microtubule-associated proteins (MAPs) have received considerable attention because they promote MT assembly and stabilization in vitro (for review, see Schoenfeld and Obar, 1994). One MAP that has been studied extensively in terms of its involvement in axon growth is tau. A role for tau in axon growth initially was suggested by the demonstration of a temporal correlation among the expression of tau, MT assembly, and axon extension (Drubin et al., 1985). More

\footnotetext{
Received May 28, 1998; revised Aug. 12, 1998; accepted Aug. 17, 1998.

This work was supported by National Institutes of Health Grants NS17681 and NS34809 (M.M.B.) and NS24275 and NS24707 (I.F.). We thank Drs. Lester Binder for providing tau and MAP2 antibodies, Greg Gundersen for providing detyrosinated tubulin antibodies, Virginia Lee for providing tau49 antibodies, and Erika Holzbaur for advice on the affinity purification of antibodies.

Correspondence should be addressed to Dr. Mark M. Black, Department of Anatomy and Cell Biology, Temple University School of Medicine, 3400 North Broad Street, Philadelphia, PA 19140.

Copyright (C) 1998 Society for Neuroscience $\quad 0270-6474 / 98 / 188660-14 \$ 05.00 / 0$
}

recently, studies that have altered tau expression in cultured neurons or neuron-like cells have reinforced the view that tau participates in axon growth. Specifically, suppressing tau expression can diminish axon growth, whereas overexpressing tau in PC12 cells can enhance axon growth (Esmaeli-Azad et al., 1994; DiTella et al., 1996).

Although the participation of tau in axon growth is well established, its specific functions are unknown. The generation of a tau knock-out mouse with little or no effect on phenotype (Harada et al., 1994) indicates that tau does not perform unique functions essential for axon growth. Because tau binds MTs, some of its functions presumably involve binding to MTs. In the test tube the principal effect of tau is to stabilize MTs by reducing catastrophe frequency (Trinczek et al., 1995). On this basis, it has been proposed that tau functions in axon growth by stabilizing MTs and thereby promoting MT assembly. Our goal in the present studies is to test this hypothesis.

We developed a protocol for acutely inactivating tau in cultured neurons by the microinjection of tau antibodies (Abs). We used a neuronal culture system in which the timing of axon initiation can be controlled, and, once initiated, axon growth proceeds vigorously (Slaughter et al., 1997). Neurons without processes were injected with tau Abs, and then they were induced to extend axons. The injected Abs quantitatively precipitated tau in the cell body. The injected neurons grew axons that contained MTs but no tau. We used this preparation to examine the effects of tau depletion on the properties of the MT array in growing axons.

\section{MATERIALS AND METHODS}

Materials

Culture media were obtained from Life Technologies (Grand Island, NY). Supplements for culture media were obtained from either Life 
Technologies or Sigma (St. Louis, MO), except for nerve growth factor, which was purified from mouse salivary glands, as described previously (Black et al., 1994). Nocodazole was obtained from Aldrich (Milwaukee, WI), and other reagents were obtained from Sigma unless otherwise indicated.

\section{Cell culture}

Dissociated cultures of rat sympathetic neurons were prepared by using modifications of our previously published procedures (Slaughter et al., 1997). These modifications permitted us to control when the neurons initiate axon growth, and, once it was initiated, axon growth proceeded relatively rapidly. Neurons were grown on glass coverslips in $35 \mathrm{~mm}$ plastic tissue culture dishes. To prepare the culture dishes, we drilled a hole $1 \mathrm{~cm}$ in diameter through the bottom of each dish, placed an acid-washed glass coverslip $(22 \times 22 \mathrm{~mm}$, number 1 thickness $)$ under the hole, and fixed it in place with a 3:1 mixture of paraffin and Vaseline (Brown et al., 1992). Neurons were dissociated from superior cervical ganglia of 1- to 3-d-old rat pups, using sequential treatments with collagenase and trypsin, followed by trituration, as previously described (Black and Kurdyla, 1983). Then the neurons were plated in serum-free medium (Brown et al., 1992) onto glass coverslips pretreated with polyL-lysine $(1 \mathrm{mg} / \mathrm{ml}$ in borate buffer). The neurons attach to this substrate relatively rapidly, but they do not extend axons for $\sim 2 \mathrm{~d}$. During this period the cells have a disk-like shape. To induce rapid axon outgrowth, we fed the neurons with medium containing $10 \%$ fetal calf serum and matrigel (Collaborative Biomedical Products, Bedford, MA), diluted 1:400 from the stock supplied by the company. In the experiments reported here, neurons were injected with tau or control Abs before the stimulation to extend axons.

\section{Affinity purification of antibodies against tau}

We previously have prepared and characterized two rabbit polyclonal Abs against tau (Black et al., 1996). The Abs were raised against recombinant fusion protein constructs containing discrete regions near the $\mathrm{N}$ terminus $\left(\operatorname{tau}^{\prime}\right)$ or $\mathrm{C}$ terminus (tau3') of the protein. The specificity of both tau Abs was demonstrated by immunoblotting against brain and culture extracts. The Abs were affinity-purified for use in microinjection experiments as follows. Purified tau, prepared from bovine brain as described previously (Black, 1987), was conjugated to activated $\mathrm{CH}$ Sepharose 4B (Pharmacia, Piscataway, NJ) according to the manufacturer's directions; $4 \mathrm{mg}$ of tau was used per milliliter of resin. The tau Abs were precipitated from serum by the addition of ammonium sulfate to $50 \%$, dissolved in PBS, and then applied to the affinity column. The column was rinsed sequentially with PBS, lithium buffer $(1 \mathrm{M} \mathrm{LiCl}, 150$ $\mathrm{mm} \mathrm{NaCl}, 10 \mathrm{~mm}$ Tris-HCl, and $0.5 \% \mathrm{NP}-40, \mathrm{pH} 8.0$ ), and then PBS. Bound $\mathrm{Ab}$ was eluted with $100 \mathrm{~mm}$ triethylamine. The eluted fractions were desalted immediately on NAP-10 columns (Pharmacia) equilibrated with injection buffer ( $50 \mathrm{~mm} \mathrm{~K}$-glutamate, $\mathrm{pH}$ 7.2). The resulting affinitypurified Abs were dialyzed against injection buffer and then concentrated with Centricon 30 concentrators (Amicon, Beverly, MA). The final material was stored in aliquots at $-80^{\circ} \mathrm{C}$. The concentration of the affinity-purified Abs was determined with the BCA assay (Pierce, Rockford, IL), using bovine serum albumin as a standard. The purified tau3' $\mathrm{Ab}$ was at $12.1 \mathrm{mg} / \mathrm{ml}$, whereas the tau5' $\mathrm{Ab}$ was at $11 \mathrm{mg} / \mathrm{ml}$. The purified Abs retained activity and specificity against tau, as determined by immunoblotting and immunostaining.

\section{Microinjection of tau Abs}

On the day of plating the cells to be injected or to serve as noninjected controls were selected, and their positions were marked on the bottom of the coverslip, using a diamond-marker objective. Selecting the cells to serve as noninjected controls before stimulating axon growth was necessary to avoid unintentional bias in selecting control cells on the basis of the morphological appearance of their axonal array. On the next morning the neurons were microinjected either with affinity-purified Abs against

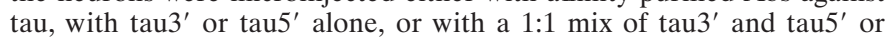
purified nonimmune rabbit IgG (Jackson ImmunoResearch, West Grove, PA); only neurons without axons were injected. Immediately before use the Abs were thawed rapidly and then clarified by centrifugation at $200,000 \times g$ for 10 min in a Beckman TL-100 ultracentrifuge (Beckman Instruments, Palo Alto, CA) to remove protein aggregates. Then the Abs were pressure-injected into cultured neurons with a Narishige micromanipulator (Tokyo, Japan), an Eppendorf injector (Hamburg, Germany), and micropipettes with a tip diameter $\leq 0.5 \mu \mathrm{m}$ [pipettes were prepared immediately before use with a Sutter Instruments P-97 pipette puller
(Novato, CA)]. To ensure that a relatively large quantity of $\mathrm{Ab}$ was injected into the cells, we used injection parameters that caused a noticeable swelling of the cell body. Generally, between 30 and $120 \mathrm{~min}$ after injection with Abs the neurons were induced to extend axons by the addition of matrigel and serum to the medium; at varying times thereafter the injected and uninjected cells were viewed with phase optics to evaluate axon growth and/or were fixed and processed for immunofluorescence localization of MTs, tau or other MAPs, and injected Ab (see below for details).

In a small number of experiments, biotin-labeled tubulin (Bt-tub) was microinjected into neurons previously injected with anti-tau Abs (for details, see Results). Bt-tub was prepared as described previously (Li and Black, 1996).

\section{Fixation and extraction of neurons}

Cultured neurons were processed for immunofluorescence localization according to one of the following procedures.

Procedure 1. Most experiments used the combined fixation and extraction procedure described by Lee and Rook (1992). Cells were rinsed once with PBS and once with PEM (80 mM PIPES, 5 mM EGTA, and 1 $\mathrm{mM} \mathrm{MgCl}_{2}, \mathrm{pH} 6.8$ ) and then were incubated with PEM containing $0.5 \%$ (w/v) IGEPAL CA-630 (Sigma), and 0.3\% glutaraldehyde (Polysciences, Warrington, PA) for $10 \mathrm{~min}$ at room temperature. After fixation the cells were rinsed with PBS and then further permeabilized by incubation with $0.5 \%$ Triton X-100 in PBS for 10 min. We have shown previously that this procedure preserves axonal MTs very well and also is superior to several other methods that have been tested with regard to providing a strong tau signal and reliably fixing assembled tau on MTs while at the same time extracting unassembled tau and tubulin from axons (Black et al., 1996). Experiments in which neurons were stained for MAP1b used a modification of this procedure in which a mix of $4 \%$ paraformaldehyde (EM Sciences, Gibbstown, NJ) plus $0.1 \%$ glutaraldehyde was used in place of $0.3 \%$ glutaraldehyde. This was necessary because the MAP1b Ab that we used (MAP5, Sigma) did not work well on cells fixed with $0.3 \%$ glutaraldehyde.

Procedure 2. Cells were fixed without extraction in PEM containing $0.3 \%$ glutaraldehyde for $10 \mathrm{~min}$ at room temperature. After fixation the cells were rinsed with PBS and then permeabilized by incubation with $0.5 \%$ Triton X-100 (in PBS) for 15 min.

Procedure 3. Neurons were extracted with $0.2 \%$ IGEPAL CA-630 in a MT-stabilizing buffer [PEM buffer containing $10 \mu \mathrm{M}$ taxol (a gift from the National Cancer Institute, Bethesda, MD) plus $0.5 \mu \mathrm{M}$ phalloidin (Sigma)]; the extraction solution also contained a mixture of protease inhibitors $(0.5 \mathrm{~mm}$ PMSF, 0.2 trypsin inhibitory $\mathrm{U} / \mathrm{ml}$ of aprotinin, and 10 $\mu \mathrm{g} / \mathrm{ml}$ each of leupeptin, chymostatin, and antipain). Extraction occurred for $5 \mathrm{~min}$ at room temperature, and then the neurons were fixed with PEM containing $0.3 \%$ glutaraldehyde for $10 \mathrm{~min}$ at room temperature. After fixation the cells were rinsed with PBS and incubated with $0.5 \%$ Triton X-100 in PBS for $10 \mathrm{~min}$.

After fixation and permeabilization the dishes were rinsed with PBS, treated with sodium borohydride $(10 \mathrm{mg} / \mathrm{ml}$ in PBS for $7 \mathrm{~min})$, incubated with $0.1 \mathrm{M}$ glycine in PBS for $20 \mathrm{~min}$, rinsed with PBS again, and then treated with blocking solution for 30 min before incubation with primary Abs. The blocking solution consisted of $10 \%$ normal donkey serum plus $10 \mathrm{mg} / \mathrm{ml}$ bovine serum albumin in PBS. Then the cells were double- or triple-stained to reveal tubulin, tau or other MAPs, and the injected Abs (see below); the cells were treated with blocking solution before incubation with all secondary Abs. All antibodies for staining were diluted in blocking solution and then clarified before use by centrifugation at $200,000 \times g$ for $10 \mathrm{~min}$ in a Beckman TL-100 ultracentrifuge (Beckman Instruments). After incubation with secondary antibodies the cells were rinsed extensively with PBS and then mounted in $80 \%(\mathrm{w} / \mathrm{v})$ glycerol in PBS containing $10 \mathrm{mg} / \mathrm{ml} n$-propyl gallate.

\section{Immunofluorescence procedures}

Cells were double- or triple-stained, using immunofluorescence procedures to reveal various antigens. Mouse monoclonal Abs against total $\alpha$-tubulin $(\mathrm{DM} 1 \alpha)$ and total $\beta$-tubulin $(\mathrm{DM} 1 \beta)$ were purchased from Amersham (Arlington Heights, IL). Mouse monoclonal Abs against acetylated tubulin (6-11B-1) and MAP1b (MAP5) were obtained from Sigma. A rat monoclonal against tyrosinated $\alpha$-tubulin, Y11/2, was obtained from Accurate Scientific (Westbury, NY). Detyrosinated tubulin was revealed with a mouse monoclonal Ab (generously provided by Dr. Greg Gundersen, Columbia University, New York, NY). Endogenous tau was revealed with the tau $\mathrm{Ab}$, a mouse monoclonal $\mathrm{Ab}$ (generously 
provided by Dr. Lester Binder, Northwestern University, Evanston, IL). The tau $1 \mathrm{Ab}$ recognizes tau only when it is not phosphorylated at the tau1 epitope. To maximize the ability of this $\mathrm{Ab}$ to reveal endogenous tau, we treated fixed cells with phosphatase, as described in Papasozomenos and Binder (1987), before incubation with antibody. Cultures that were fixed, extracted, and blocked as described above were rinsed with $0.1 \mathrm{M}$ Tris- $\mathrm{HCl}, \mathrm{pH} 8.0$, and incubated at $37^{\circ} \mathrm{C}$ for $3-4 \mathrm{hr}$ with $0.5 \mathrm{mg} / \mathrm{ml}$ of type VII-L alkaline phosphatase from bovine intestine (Sigma) in 0.1 $\mathrm{M}$ Tris-HCl, $\mathrm{pH} 8.0$, containing a cocktail of protease inhibitors (see above). After this treatment the cultures were rinsed with cold $0.05 \mathrm{M}$ Tris- $\mathrm{HCl}$ plus $0.2 \mathrm{M} \mathrm{NaCl}, \mathrm{pH} 7.4$, and then stained as described below. This phosphatase treatment effectively dephosphorylates the endogenous tau so that most or all of it can be revealed with the tau $1 \mathrm{Ab}$. In some experiments tau49, a phosphorylation-independent mouse monoclonal Ab against tau (generously provided by Dr. Virginia Lee, Department of Pathology, University of Pennsylvania, Philadelphia, PA), also was used to reveal endogenous tau. All fluorescent secondary Abs were purchased from Jackson ImmunoResearch (AffiniPure grade, preadsorbed for minimum cross-reactivity with other species). Biotin-labeled anti-mouse Abs and cy2-labeled streptavidin were purchased either from Jackson ImmunoResearch or Amersham.

Triple staining was performed to visualize endogenous tau, tyrosinated tubulin, and the injected $\mathrm{Ab}$ in the same cells. For triple staining, cells that were fixed and processed according to Procedure 1 (see above) were incubated with tau1 alone or a mix of tau 1 and tau49, rinsed twice with PBS, incubated with the rat monoclonal against tyrosinated tubulin, rinsed extensively with PBS, incubated with biotin-labeled donkey antimouse secondary Ab, rinsed extensively with PBS, and then incubated simultaneously with cy2-labeled streptavidin, lissamine-labeled donkey anti-rat Abs, and cy5-labeled donkey anti-rabbit Abs, all at 1:100 dilution. After being rinsed extensively, the cells were mounted and coverslipped. Triple staining also was used to reveal tyrosinated MTs, injected Ab, and either MAP1b or MAP2. These procedures were performed as described above, except that mouse monoclonal Abs against MAP2 (AP14, a generous gift from Dr. Lester Binder, or clone HM2 from Sigma) or MAP1b (anti-MAP5, Sigma) were used in place of the tau Abs.

Triple staining also was used to visualize MTs, tau, and actin filaments. For this purpose the cells were incubated with primary Abs against tau and tyrosinated tubulin as described above. Then, after being rinsed extensively, the cells were incubated with biotin-labeled donkey antimouse secondary $\mathrm{Ab}$, rinsed extensively with PBS, and finally incubated simultaneously with cy2-labeled streptavidin, cy5-labeled donkey anti-rat Abs, and rhodamine-labeled phalloidin (Sigma). After being rinsed extensively, the samples were mounted as described above.

Double-staining procedures were used to stain for MTs, using mouse monoclonal antibodies against tubulin, and the injected Abs. These analyses used cells extracted before fixation by using Procedure 3 described above. Cells were incubated with the tubulin Ab, rinsed extensively, and then incubated with cy2-labeled donkey anti-mouse and lissamine-labeled donkey anti-rabbit secondary Abs. After being rinsed extensively, the cells were mounted as described above.

In experiments microinjecting both Bt-tub and anti-tau Abs, the cells were processed according to Procedure 3 as described above. The cells were stained with Rh-phalloidin to reveal actin filaments, cy5 donkey anti-rabbit to reveal the injected $\mathrm{Ab}$, and a mouse monoclonal $\mathrm{Ab}$ against biotin (Jackson ImmunoResearch), followed by cy2-labeled donkey antimouse, to reveal Bt-tub in MTs.

\section{Image acquisition}

In most experiments the neurons were observed by epifluorescence microscopy with a Zeiss Axiovert 135 inverted microscope (Carl Zeiss, Thornwood, NY), and images were obtained with a $\mathrm{CH} 250$ cooled charge-coupled device (CCD) camera (Photometrics, Tucson, AZ) equipped with a Thompson 7883 CCD chip. The details of the imaging system have been described previously (Brown et al., 1992; Black et al., 1994; Li and Black, 1996). Images were acquired by using the full usable area of the CCD chip, which measured $382 \times 576$ pixels, and were stored in full 12-bit format on magneto-optical disks, using Pinnacle optical disk drives (Pinnacle Micro, Irvine, CA). Before a series of images was captured, an instantaneous readout of the bias voltage offset on the chip was saved and subsequently was subtracted from each exposed image. Dark current ( 0.133 analog-to-digital U/sec) was not significant for the exposure times used in these studies. The magnification of the CCD images was calibrated with a stage micrometer. For maximum precision, all measurements of fluorescence intensity (see below) were performed on the 12-bit images. For presentation the images were scaled to eight bits, saved in tagged image file format (TIFF), and then imported into Adobe Photoshop to compose the figures; text and arrowheads were added with Adobe Illustrator.

For some experiments the cells were imaged with a Zeiss confocal microscope, using a $40 \times$ magnification, 1.3 numerical aperture (NA) Plan oil-immersion objective. $Z$-steps were at $0.4 \mu \mathrm{m}$, and a sufficient number of sections was taken to include the entire thickness of the cell body.

To measure axon growth rates, we observed control and injected cells by phase-contrast microscopy with a Zeiss Axiovert 35 inverted microscope and a $10 \times$ magnification, 0.3 NA Plan Neofluar or a $20 \times$ magnification, $0.45 \mathrm{NA}$ or $32 \times$ magnification, 0.4 NA achrostigmat objective, together with a 0.3 NA condenser (Zeiss). Images of cells were captured approximately every $90 \mathrm{~min}$ with a Newvicon camera (Hamamatsu Photonic Systems, Bridgewater, NJ) interfaced to an Apple Quadra 950 computer with a pixel pipeline framegrabber board (Perceptics, Knoxville, TN) and the Oncor Imaging processing and analysis software package (Rockville, MD). Axon lengths were determined on the basis of a one-pixel-wide line drawn down the center of the axon, using programs written in our laboratory with the Oncor Imaging programming language. Growth rates then were calculated from the change in length divided by the change in time.

\section{Image analysis}

To quantify the relative amount of tubulin and tau in axons of injected or uninjected cells, we imaged cells with a $40 \times$ magnification, 1.3 NA Plan Neofluar oil-immersion objective. To examine microtubule staining for tubulin and tau at higher resolution, we imaged cells either with a $63 \times$ magnification, 1.4 NA Plan apochromatic oil-immersion objective or a $100 \times$ magnification, 1.3 NA Plan Neofluar oil-immersion objective. We used the segmented mask procedure (Brown et al., 1992) to quantify the intensity of tubulin and tau staining along the length of individual axons in images like those shown in Figure $1, B$ and $C$. For these analyses we focused primarily on the distal $50 \mu \mathrm{m}$ of the axon, because this is the region of the growing axon where tau is most abundant and where the amount of tau on MTs is also the highest (Black et al., 1996). Briefly, the tubulin image was used to generate a mask of the axon that extended from the cell body to the tip of the axon. The mask was divided into consecutive $\sim 5-\mu \mathrm{m}$-long segments beginning at the axon tip and extending toward the cell body. Then the segmented mask was overlaid on the original fluorescent images depicting the distribution of tau and tubulin. This allowed us to define segments in the fluorescent images that corresponded to the segments in the mask. To correct for background fluorescence in each image, we calculated an average background pixel intensity for each segment and subtracted it from each pixel within that segment. Then the total fluorescence intensity for the segments in each image was calculated by summing the corrected intensities of the pixels comprising each segment. These procedures have been incorporated into a single interactive application program written in the Oncor Imaging programming language. A synopsis of these procedures is presented in Brown et al. (1992), and a more detailed description will be provided on request. To quantify the staining intensity for tubulin and tau in the distal $50 \mu \mathrm{m}$ of the axon, we summed the fluorescence intensity in the distal 10 segments. Statistical comparison of the staining in axons of injected and uninjected cells was performed with StatView software (Abacus Concepts, Berkeley, CA), using the unpaired Student's $t$ test and the MannWhitney $U$ nonparametric test (because similar results were obtained with both tests, the data are presented on the basis of the Student's $t$ test).

\section{RESULTS}

\section{Microinjection of tau antibodies precipitates tau in the cell body}

To examine possible functions of tau in growing neurons, we have developed a procedure that acutely inactivates tau in neurons by Ab-induced precipitation. Neurons without processes are injected with affinity-purified polyclonal Abs against recombinant tau, and then the neurons are stimulated to extend axons by the addition of matrigel and serum to the medium (see Materials and Methods). At varying times thereafter the neurons are examined to reveal the consequences of $\mathrm{Ab}$ injection on the localization of tau, on the MT array in the axon, and on axon growth. 

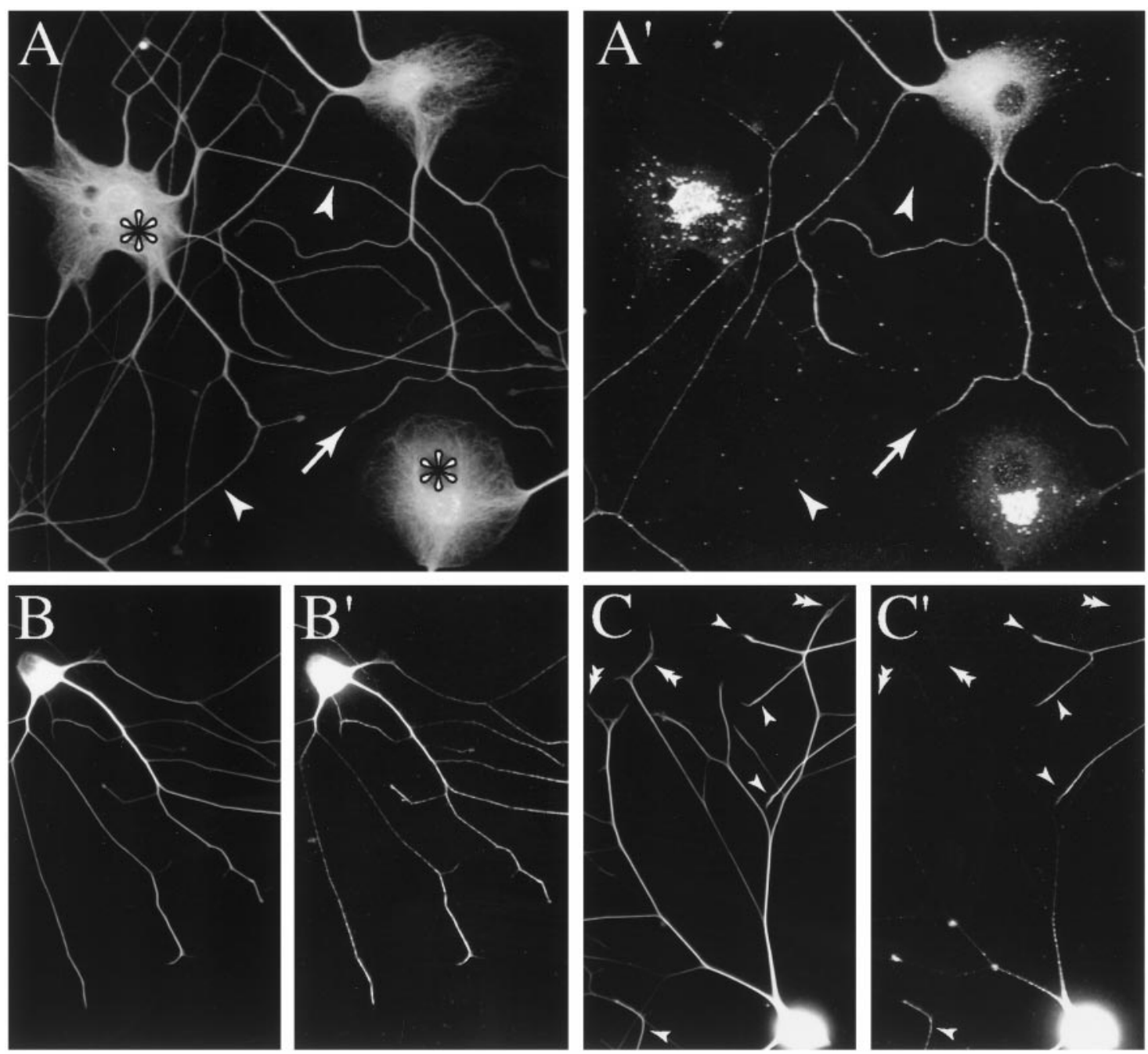

Figure 1. Distribution of MTs and tau in injected and uninjected cells. $A, A^{\prime}$, Confocal images showing the distribution of MTs $(A)$ and tau $\left(A^{\prime}\right)$ in injected and uninjected cells. The images contain two injected cells (indicated with asterisks in $A$ ) and one uninjected cell; the arrowheads point to axons of an injected cell, whereas the arrow points to an axon of the uninjected cell. Contrast and brightness have been enhanced to best illustrate cell body staining. The cells were incubated for $\sim 6 \mathrm{hr}$ after axon growth was induced and then fixed according to Procedure 1 and stained to reveal MTs and tau. Injected and uninjected cells have similar patterns of MT staining, whereas the pattern of tau staining in injected cells differs dramatically from that in uninjected cells (for details, see Results). $B, B^{\prime}, C, C^{\prime}$, Conventional low-magnification images of MT and tau distribution in an uninjected cell ( $B, B^{\prime}$ ) and a cell injected with tau $\mathrm{Abs}\left(C, C^{\prime}\right)$. The cells were incubated for $\sim 5 \mathrm{hr}$ after axon growth was induced and then fixed according to Procedure 1 and stained to reveal MTs and tau. MT staining for tyrosinated tubulin is shown in $B$ and $C$, whereas tau staining is shown in $B^{\prime}$ and $C^{\prime}$. Contrast and brightness have been enhanced to best illustrate axonal staining; as a result, cell body staining appears saturated. The single arrowheads in $C$ and $C^{\prime}$ identify axons of uninjected cells for which cell bodies are located outside the field. The double arrowheads in $C$ and $C^{\prime}$ identify axons of the injected cell. Note that MT staining extends throughout the axons of injected and uninjected cells. Tau staining in uninjected cells also extends throughout the entire axon and exhibits a typical proximal-to-distal gradient, reaching peak staining distally near the growth cone. By contrast, in injected cells tau is seen only in the cell body and proximal axon, where it occurs in aggregates.

The utility of this approach depends on the extent to which tau can be immunodepleted by the injection of tau Abs. Figure 1 shows confocal and conventional images of Ab-injected and control neurons 5-6 hr after axon growth is induced. Both types of cells have extended elaborate axonal processes (detailed analyses of axon growth behavior are presented below). By visual inspection, MT staining is indistinguishable in control and Ab-injected cells. The cell bodies stain strongly, and in more peripheral lamellar regions the staining is clearly filamentous (Fig. $1 A$ ). MT staining also extends throughout the axon and into the growth cone (Fig. $1 A-C$ ). In contrast to the situation for MTs, tau staining is very different in control versus Ab-injected cells. In the former, the cell body stains diffusely for tau, the axons stain all along their length, and most axons exhibit a pronounced proximal-to-distal increase in the intensity of tau staining (Fig. $\left.1 A^{\prime}, B^{\prime}\right)$ (Black et al., 1996). In Ab-injected cells, tau staining differs from that of controls in two major respects. First, in the cell body, tau staining reveals aggregate-like structures localized principally to the central region, where the cell body is thickest; this is best appreciated in confocal images (Fig. $1 A^{\prime}$ ). In addition to this centrally located staining, some injected cells also have discrete foci of tau staining scattered in the soma and proximal axon. Second, little or no tau staining can be detected in the axons, other than the discrete aggregates of tau staining present proximally (Fig. $1 A^{\prime}, C^{\prime}$ ). Our interpretation of these data is that the injected $\mathrm{Ab}$ immunoprecipitates tau in the cell body into large aggregates, thereby sequestering tau so that it cannot diff use or be transported into the axon. This interpretation is supported by the 
following two observations. First, the injected $\mathrm{Ab}$ is concentrated preferentially at the same sites as the tau in Ab-injected cells, although overall it has a broader distribution than tau, extending throughout the cell body and axon (data not shown). Second, in control neurons, tau is removed quantitatively during extraction with MT stabilizing buffers that contain nonionic detergents (Black et al., 1996). However, in Ab-injected cells the somal staining of tau aggregates persists after such extractions (data not shown).

Microinjections of the tau3' Ab alone or a 1:1 mix of the tau3' and tau5' Abs were both very effective at aggregating tau in the cell body and preventing it from entering the axon. The tau5' Ab alone was not as effective as tau3' alone or the mix of the two Abs in precipitating tau in the cell body or in reducing axonal staining for tau. The results reported here were obtained by using tau3' alone or a mix of the two Abs.

The effectiveness with which tau is sequestered from the axon can be appreciated by high-magnification views of tau staining in the distal axon and growth cone regions of control and Abinjected cells. In control neurons the strongest axonal staining for tau is seen distally near the growth cone (Figs. 1, 2B) (Black et al., 1996). However, in Ab-injected cells only a hint of glow is apparent in this region (Fig. $2 C-F$ ). This glow reflects principally nonspecific staining, because it is comparable in intensity to that seen in the thin peripheral regions of non-neuronal cells in the cultures (Fig. 2C,D), which do not express tau. Thus, the Ab injection procedure very effectively sequesters tau in the cell body and thereby prevents it from entering the axon.

To quantify the extent to which the injection of tau Abs reduces the amount of tau present in the distal axon, we compared the intensity of tau staining in the distal $50 \mu \mathrm{m}$ of axons of control and Ab-injected cells (for details, see Materials and Methods). Results from a representative experiment are shown in Figure 3. The staining intensity in control axons is quite variable, reflecting, in part, the morphological heterogeneity (principally thickness) of the axons. However, in all cases the staining intensity in control axons exceeds that seen in axons of Ab-injected cells. In this regard, note that the maximum fluorescence observed in the distal axon of Ab-injected cells is substantially less than the minimum staining observed in the control axons. In six separate experiments, three that involved injecting the mix of tau Abs and three that involved injecting just tau3', staining in Ab-injected cells was $11 \pm 3 \%$ (mean $\pm \mathrm{SD}$ ) of that in uninjected cells, and in all six experiments the results obtained with anti-tau-injected cells were significantly different from uninjected cells at $p<$ 0.0001 . This reduction in tau staining depends on the injection of tau Abs. Control experiments in which nonimmune Ab was microinjected had no significant effect on axonal staining for tau. Specifically, tau staining in the distal $50 \mu \mathrm{m}$ of cells injected with nonimmune $\mathrm{Ab}$ was $113 \pm 19 \%(n=4)$ of that in control cells. Furthermore, there was no indication that the injection of tau Abs caused an aggregation of proteins other than tau. For example, tubulin, MAP2, and MAP1b did not coaggregate with tau in the Ab-injected cells (see Fig. 1) (data not shown).

These quantitative analyses on tau staining in control neurons and neurons injected with tau Abs did not take into account the amount of nonspecific staining present in our material. An indication of the nonspecific staining can be obtained by examining non-neuronal cells present in the cultures (see Fig. 2), because these cells do not express tau. In control axons, nonspecific staining represents only a small portion of the specific staining. However, the glow associated with axons of anti-tau-injected cells is very similar to that associated with non-neuronal cells, indicating that nonspecific staining accounts for most, if not all, of the glow associated with axons of Ab-injected cells. Thus, the injection of tau Abs reduces the amount of tau in the distal axons by much more than $90 \%$. These considerations indicate that the $\mathrm{Ab}$ injection approach used here effectively sequesters tau to the cell body. Under these conditions the cells extend axons, but the axons contain little if any tau. We have taken advantage of this model system to examine the effects of acute tau removal on the properties of axonal MTs.

\section{Immunodepletion of tau has no measurable affect on the amount of MT polymer in growing axons}

To determine whether tau affects MT assembly and/or stability in growing axons, we first addressed whether axons formed without tau differ from control axons in their content of MT polymer. By visual inspection, the cells injected with tau Abs extend axons that have a relatively normal-appearing MT array. MT staining is continuous from the cell body to the growth cone of control and Ab-injected cells, and the intensity of axonal staining for MTs is not obviously different between the two groups of cells (see Figs. $1,2)$. These considerations suggest that the immunodepletion of tau has minimal effect on the amount of axonal MT polymer, and quantitative analyses confirm this interpretation. We measured the intensity of MT staining in the distal $50 \mu \mathrm{m}$ of axons of control and Ab-injected cells. Data from two experiments are shown in Figure 4. The staining intensity of MTs varies over a broad range from one axon to another. However, the distributions of values for control and Ab-injected cells are very similar to each other; statistical analyses indicate that the values in the two groups of cells are not different from each other (for details, see legend to Fig. 4). In a total of three experiments in which tau Abs were injected, MT staining in Ab-injected cells was 92-96\% of that in uninjected control neurons. In three experiments in which nonimmune $\mathrm{Ab}$ was injected, MT polymer levels in injected cells were $112 \pm 4 \%$ of those in control cells, and statistical analyses indicate that the values obtained from the cells injected with nonimmune $\mathrm{Ab}$ are not different from those obtained from uninjected cells.

We also compared details of the MT array in growth cones of $\mathrm{Ab}$-injected and control neurons. For these comparisons we focused on those growth cones that had a well spread morphology, because the MTs splay out from each other, making it possible to visualize individual MTs and small bundles of MTs by immunofluorescence procedures. On the basis of visual inspection, spread growth cones of control and Ab-injected cells appeared to have a comparable abundance of MTs, with the MTs extending throughout much of the volume of the growth cone (see Fig. 2). Also, the relative straightness or waviness of MTs in growth cones of Ab-injected cells resembled that of control neurons. Thus, by qualitative as well as by quantitative analyses, the immunodepletion of tau had no detectable effect on the amount of MT polymer present in growing axons or their growth cones.

\section{Post-translational modifications of axonal MTs are not detectably affected by the immunodepletion of tau}

Although overall MT polymer levels appear to be unaffected by tau inactivation, it is possible that the dynamic behavior of axonal MTs would be altered, especially given the well documented effects of purified tau on MT dynamics in the test tube. To evaluate this possibility, we examined the relative amounts of 


\section{Microtubules}
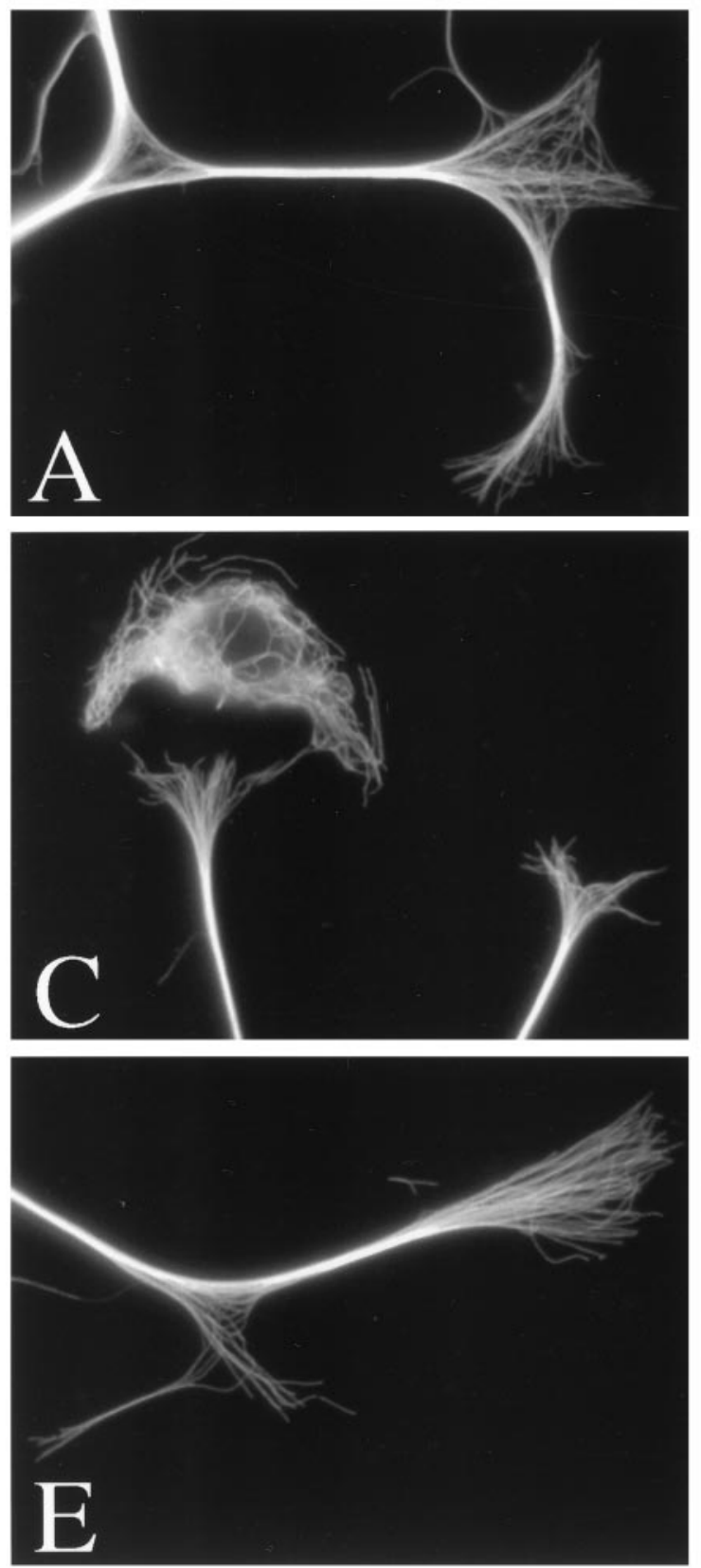

Tau
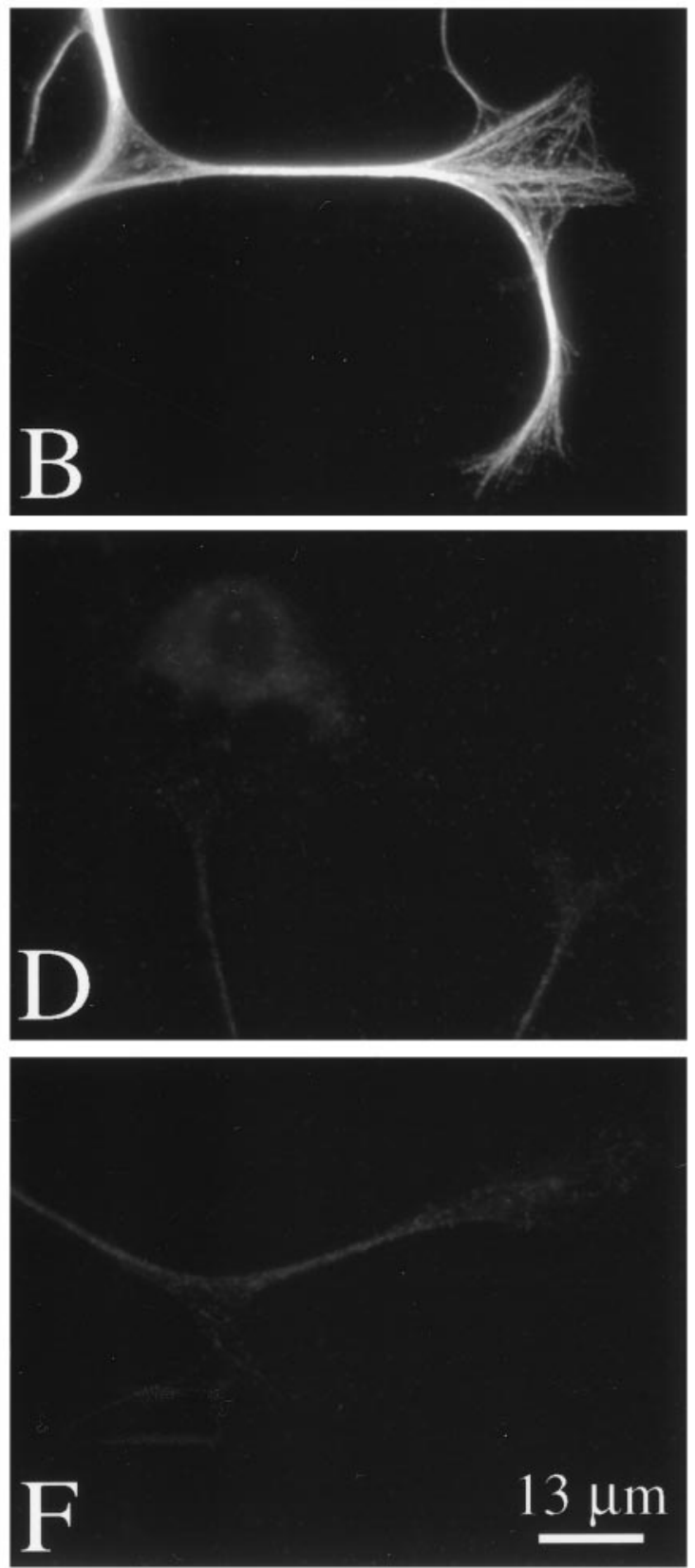

Figure 2. High-magnification images of MT and tau staining in the distal axon and growth cones of an uninjected cell $(A, B)$ and two different cells that were injected with tau Abs $(C-F)$. The cells were incubated for $\sim 6 \mathrm{hr}$ after axon growth was induced and then fixed according to Procedure 1 and stained to reveal tyrosinated tubulin in MTs $(A, C, E)$ and tau $(B, D, F)$. Note the abundance of MTs in growth cones of control and injected cells. In growth cones of control cells, MTs are decorated strongly with tau along most of their length. By contrast, MTs in growth cones of injected cells do not stain detectably for tau. Note the non-neuronal cell present in the images of injected cells $(C, D)$. These cells do not contain tau and thus provide an indication of background glow in the tau images. The dim fluorescence associated with the injected axons stained for tau is comparable to that seen in non-neuronal cells, indicating that this fluorescence is attributable principally to background.

various post-translationally modified tubulins in axonal MTs of control and Ab-injected cells. Several studies have shown that the relative abundance of tyrosinated (tyr) tubulin, detyrosinated (detyr) tubulin, and acetylated (Ac) tubulin in a MT correlates with its stability properties such that tyr-tubulin is especially enriched in the more dynamic MT polymer of cells, whereas detyr-tubulin and Ac-tubulin are especially enriched in the more stable MT polymer of cells (for review, see Brown et al., 1992).
This correlation is especially strong in growing axons, in which the more dynamic polymer is rich in tyr-tubulin but poor in detyrand Ac-tubulins, whereas the more long-lived polymer is poor in tyr-tubulin but rich in detyr- and Ac-tubulins (Baas and Black, 1990; Brown et al., 1993; Li and Black, 1996). In the present experiments we have measured the relative amount of tyrtubulin, detyr-tubulin, and Ac-tubulin in axonal MTs of control and $\mathrm{Ab}$-injected cells as a means to evaluate whether the immu- 


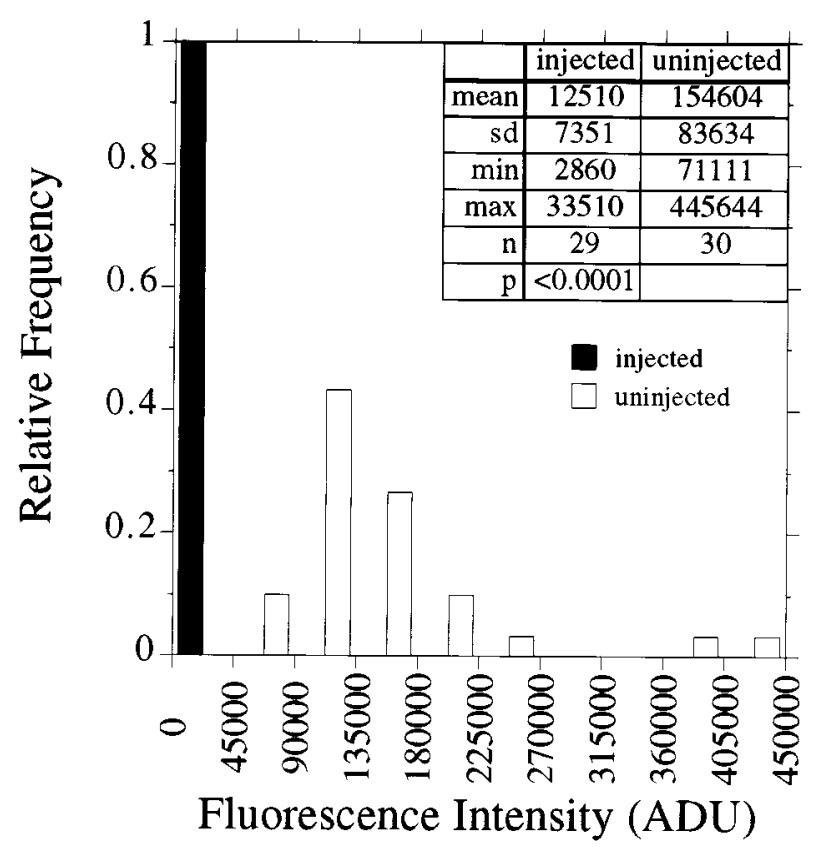

Figure 3. Quantitative analyses of tau in axons of control and Abinjected neurons. The fluorescence intensity caused by tau staining was quantified in the distal $50 \mu \mathrm{m}$ of axons of control cells and cells that were injected with tau Abs; analyses were performed on images like those depicted in Figure 1, $B$ and $C$. Cells were processed according to Procedure 1 (see Materials and Methods). Shown is a frequency distribution of the fluorescence intensity values that were obtained. The data presented are from a single experiment that is representative of several similar experiments. The distribution of values for control cells is relatively broad, but it is totally different from that for Ab-injected cells. In this experiment the injection of tau Abs reduced tau in the distal axon by at least $92 \%$.

nodepletion of tau alters the overall dynamic behavior of the axonal MT array.

Figure 5 shows the results of an analysis of the relative amounts of tyr-tubulin and detyr-tubulin in MTs in the distal $50 \mu \mathrm{m}$ of axons of control versus Ab-injected cells, and data from three replicate experiments are shown in Table 1 . The relative amounts tyr-tubulin and detyr-tubulin in the distal $50 \mu \mathrm{m}$ of the axon varied considerably in the population of control and Ab-injected axons that were examined. The immunodepletion of tau had no detectable effect on the range or magnitude of the values that were obtained. Entirely similar results also were obtained in separate experiments comparing Ac-tubulin levels in distal axons of control and Ab-injected cells (Fig. 6, Table 1). In other analyses we determined that the immunodepletion of tau also had no detectable effect on the relative amount of tyr-tubulin, detyrtubulin, and Ac-tubulin in proximal regions of the axon. Collectively, these data indicate that inactivation of tau had no measurable effect on the overall dynamic behavior of the axonal MT array.

\section{Sensitivity of axonal MTs to Nocodazole is not detectably affected by the immunodepletion of tau}

To evaluate whether the immunodepletion of tau alters MT sensitivity to Nocodazole, we measured the effect of a brief ( 5 min) treatment with Nocodazole on the loss of MT polymer from the distal part of the axon contiguous with the growth cone. The polymer in this region is relatively enriched in tau (see Fig. 3) (Black et al., 1996) and is also relatively labile to treatment with
Nocodazole, declining in amount with a half-time of $\sim 3$ min (Baas et al., 1993). This decline manifests itself as an increase in the distance between the distal-most site of axonal staining for MTs and the end of the axon. In control neurons the MT array extends into the growth cone to within 1-2 $\mu \mathrm{m}$ of its most distal extent (excluding filopodia). After short treatments with Nocodazole, the MT array frequently does not extend into the growth cone, reflecting the drug-induced loss of MT polymer from this region of the axon (Baas et al., 1993). In the present experiments we determined whether the immunodepletion of tau enhances the Nocodazole-induced increase in the distance between the distal extent of MT staining and the tip of the axon.

Control and $\mathrm{Ab}$-injected neurons were incubated with matrigel plus serum for $\sim 5 \mathrm{hr}$, Nocodazole was added to the culture medium to $5 \mu \mathrm{g} / \mathrm{ml}$, the cells were incubated for a further $5 \mathrm{~min}$, and then they were processed by using the combined fix and extraction method (Procedure 1, Materials and Methods). This method removes unassembled tubulin and tau from the axons so that the tubulin staining remaining in such cells is attributable to MTs (Black et al., 1996). After the cells were stained for tubulin and tau, fluorescence and phases images of the cells were obtained, and the distance between the distal extent of MT staining and the tip of the axon, identified with the fluorescent and phase images, respectively, was measured. In control axons the distance was $1.8 \pm 2.5 \mu \mathrm{m}$ (mean $\pm \mathrm{SD}$; range, $0-10.2 \mu \mathrm{m} ; n=34)$. In uninjected cells treated with Nocodazole the distance was $9.3 \pm$ $6.5 \mu \mathrm{m}$ (range, $0-21 \mu \mathrm{m} ; n=40$ ), whereas in injected cells treated with Nocodazole the distance was $8.7 \pm 7 \mu \mathrm{m}$ (range, $0-23 ; n=$ 33). Statistical analyses indicate that both drug-treated samples are significantly different from controls at $p<0.01$, although the two drug-treated groups are not different from each other $(p>$ 0.1 ). Thus, the immunodepletion of tau had no detectable effect on the extent to which MT polymer was lost from the distal part of the axon during a brief treatment with Nocodazole.

\section{Microtubule regrowth during recovery after treatment with Nocodazole is not affected by the injection of anti-tau Abs}

As another probe of the possible effects of the immunodepletion of tau on MT dynamics, we examined MT regrowth during recovery from treatment with Nocodazole. For these experiments the cells were treated with $5 \mu \mathrm{g} / \mathrm{ml}$ Nocodazole for $\sim 2 \mathrm{hr}$ before MT regrowth was initiated. Although this drug treatment resulted in substantial MT depolymerization, a portion of the MT polymer in axons persisted, making it difficult to evaluate MT regrowth on the basis of the assembly of endogenous tubulin. The simple approach of treating longer with drug to get more MT depolymerization was not satisfactory, because such treatments resulted in extensive axon retraction. Thus, we used Bt-tub to visualize MT regrowth, using the following experimental design. Neurons were injected with tau Abs and then induced to extend axons, as described in Materials and Methods. At 3-6 hr later, Nocodazole was added to $5 \mu \mathrm{g} / \mathrm{ml}$. At $30 \mathrm{~min}$ later, Bt-tub was injected into Ab-injected cells and into uninjected cells, and the cells were incubated an additional 90-120 min. Preliminary experiments established that the assembly of Bt-tub into MTs did not occur during the incubation in Nocodazole (data not shown). To examine MT regrowth, we rinsed the cultures with medium to remove Nocodazole, incubated them a further 10-15 min, extracted them according to Procedure 3 to remove unassembled tubulin, and then fixed the cultures. We specifically used relatively short recovery times to accentuate possible differences in re- 

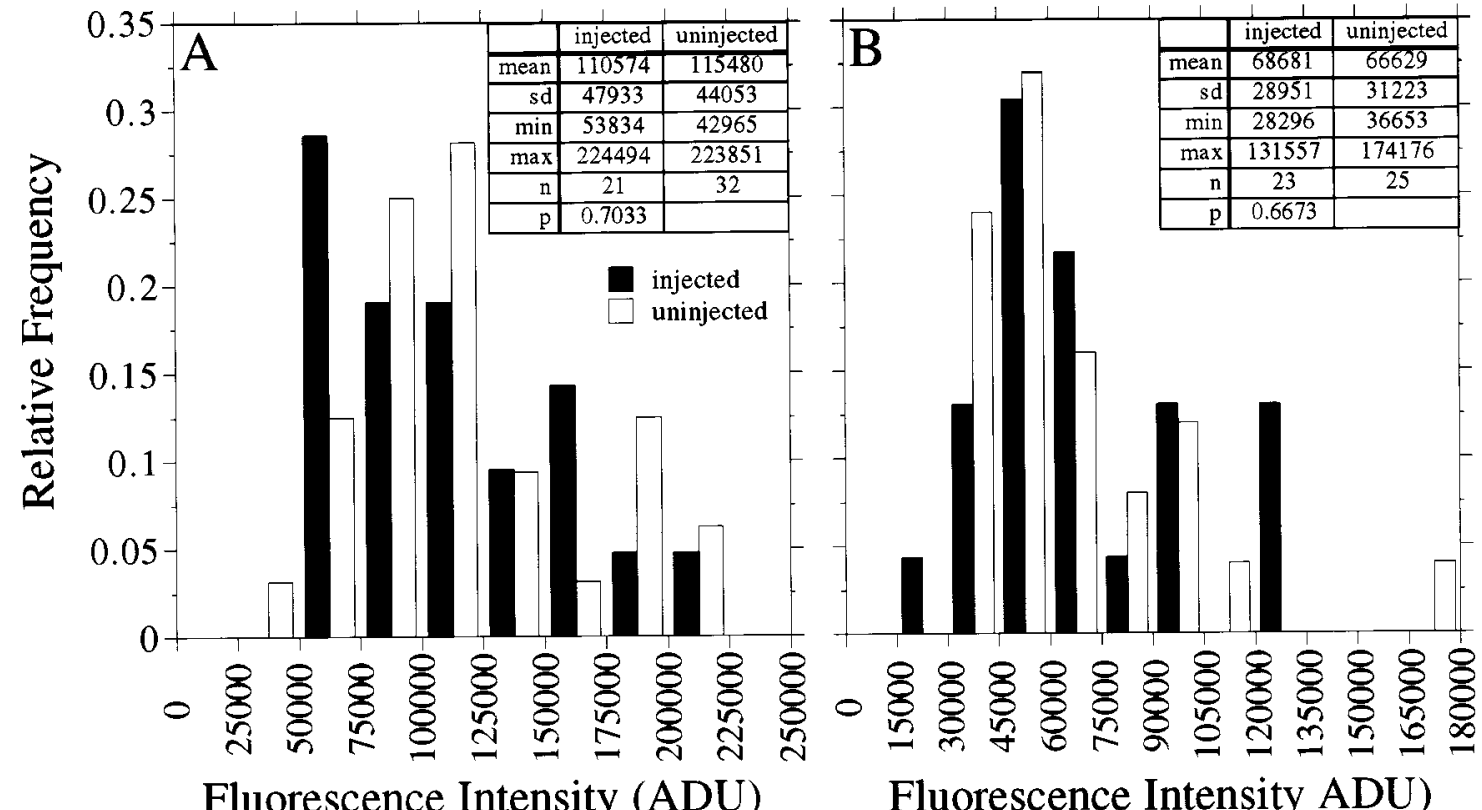

Fluorescence Intensity ADU)

Figure 4. Quantitative analyses of microtubule polymer in axons of control and Ab-injected neurons. The fluorescence intensity caused by tubulin staining was quantified in the distal $50 \mu \mathrm{m}$ of axons of control cells and cells that were injected with tau Abs, and the resulting values are displayed as a frequency histogram. The cells were processed according to Procedure 3 (see Materials and Methods). The data in $A$ were generated by using a monoclonal $\mathrm{Ab}$ against total $\beta$-tubulin, whereas the data in $B$ were generated by using a monoclonal Ab against total $\alpha$-tubulin. Statistical analyses indicated that tubulin staining in Ab-injected cells is not different from that of uninjected cells $(p>0.1)$.

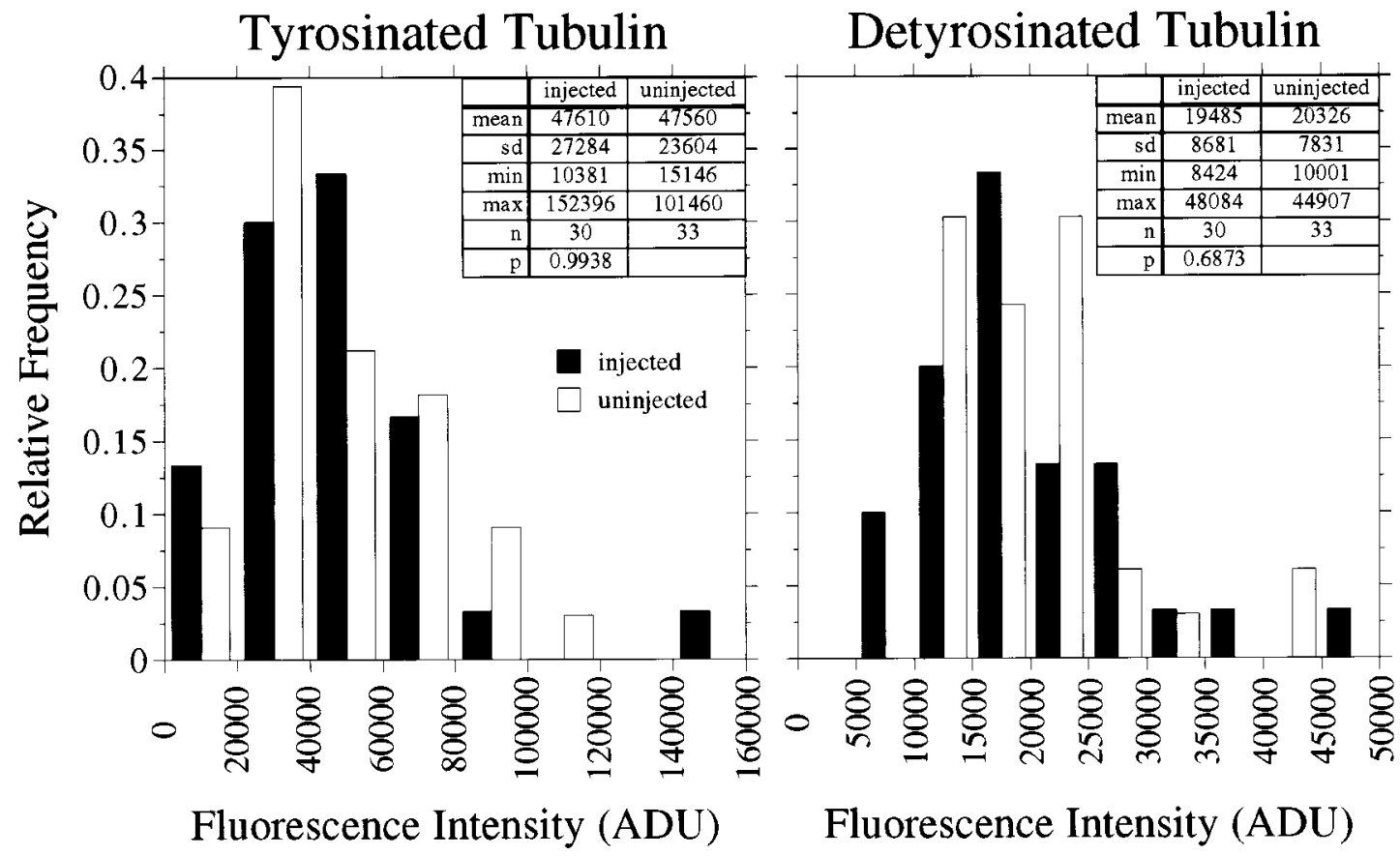

Figure 5. Quantitative analyses of tyrosinated and detyrosinated tubulin in axonal microtubules of uninjected cells and neurons that were injected with tau Abs. Control and injected cells were processed according to Procedure 3 (see Materials and Methods) and then stained to reveal tyrosinated and detyrosinated tubulin in axonal MTs. The fluorescence intensity caused by tyrosinated and detyrosinated tubulin was quantified in the distal $50 \mu \mathrm{m}$ of the axons, and the resulting values are displayed as frequency histograms. The left panel depicts data for tyrosinated tubulin, whereas the right panel depicts data for detyrosinated tubulin. The data presented are from a single experiment, and summary data from all similar experiments are presented in Table 1 . The injection of tau Abs had no statistically significant effect on the levels of tyrosinated and detyrosinated tubulin in axonal MTs.

growth that might result from the immunodepletion of tau. Then the cells were triple-stained to reveal Bt-tub, injected Ab, and actin filaments, as described in Materials and Methods. Actin staining was used to provide an image of the overall shape of the axon. Staining for the injected $\mathrm{Ab}$ provided an indirect indication of the degree of tau immunodepletion (for details, see legend to Table 1); all Ab-injected cells examined in these experiments displayed evidence of the immunodepletion of tau. 
Table 1. Comparisons of the relative amounts of tyr-tubulin, detyrtubulin, and Ac-tubulin in MTs of axons of uninjected and anti-tauinjected cells

\begin{tabular}{lcll} 
& $\begin{array}{l}\text { Tyr-tubulin (as } \\
\text { a percentage of } \\
\text { uninjected cells) }\end{array}$ & $\begin{array}{l}\text { Detyr-tubulin (as } \\
\text { a percentage of } \\
\text { uninjected cells) }\end{array}$ & $n^{*}$ \\
\hline Experiment 1 & 86 & 122 & $12 / 19$ \\
Experiment 2 & 100 & 96 & $30 / 33$ \\
Experiment 3 & 78 & 66 & $22 / 26$ \\
\hline
\end{tabular}

\begin{tabular}{llll} 
& $\begin{array}{l}\text { Tyr-tubulin (as } \\
\text { a percentage of } \\
\text { uninjected cells) }\end{array}$ & $\begin{array}{l}\text { Ac-tubulin (as } \\
\text { a percentage of } \\
\text { uninjected cells) }\end{array}$ & $n^{*}$ \\
\hline Experiment 1 & 105 & 84 & $20 / 29$ \\
Experiment 2 & 95 & 86 & $28 / 34$ \\
\hline
\end{tabular}

In these experiments it was not possible to stain for endogenous tau because the Abs available for this were mouse monoclonal, and the Abs against detyr-tubulin and Ac-tubulin were also from mouse. Thus we could not evaluate directly the effectiveness of tau sequestering in these experiments. However, in other experiments in which we stained $\mathrm{Ab}$-injected cells for endogenous tau and the injected $\mathrm{Ab}$, the large tau aggregates in the cell bodies also stained strongly for the injected Ab. Furthermore, the presence of a large tau aggregate in the cell body always correlated with the absence of tau staining in the distal axon. So, in the present experiments we evaluated the degree of tau sequestering indirectly by examining the Ab-injected cells for the presence of large aggregates of injected Ab in the cell body. The cells were extracted before fixation according to Procedure 3 as described in Materials and Methods and then were stained for the injected Abs as well as for the indicated tubulins. The extraction procedure removes most of the injected $\mathrm{Ab}$ not associated with the large tau aggregates in the cell body and proximal axon (data not shown), making the aggregates especially easy to visualize. All Ab-injected cells analyzed for these experiments had large aggregates of injected $\mathrm{Ab}$ in the cell body.

*Number of axons analyzed, injected/uninjected. Tyr-tubulin levels were compared in control and $\mathrm{Ab}$-injected cells in two other experiments in which the cells were double-stained for tyrosinated tubulin and endogenous tau. In these experiments the amount of tyr-tubulin in MTs in injected axons was 135 and $97 \%$ of that in uninjected axons. The amount of tau in the injected axons was 7 and $11 \%$, respectively, of that in the controls. The mean \pm SD of tyr-tubulin in injected cells as a percentage of that in control cells for all experiments injecting tau Abs $=99 \pm 18$ $(n=7)$. The values obtained from anti-tau-injected cells were not significantly different from uninjected cells at $p>0.1$ (except for the data for tyr-tub in Experiment 3, in which $p=0.0909$ ).

MT regrowth occurred in both control and Ab-treated cells. Recovery was evident as the appearance of segments of Bt-tub staining that appeared along the axon (Fig. 7). The degree of recovery was variable in several respects. First, the number of Bt-tub-containing MT segments was variable from one axon to another. Second, the segments were of variable length within individual axons as well as between axons. Finally, the segments were scattered along the length of the axon, with sizable portions of the axon lacking detectable assembly of Bt-tub into MTs. All of these factors made it difficult to quantify recovery in any meaningful way. On the basis of visual inspection of several control and Ab-injected cells, we had the impression that the extent of recovery was comparable in control and Ab-injected cells (Fig. 7) and, therefore, that the immunodepletion of tau did not impair MT regrowth substantially during recovery after treatment with Nocodazole.

\section{Effects of tau inactivation on other properties of the axonal cytoskeleton}

Tau is only one of several MAPs present in these neurons (Peng et al., 1985). We evaluated whether the immunodepletion of tau altered the distribution of other MAPs, focusing on MAP2 and MAP1b because they are relatively abundant in these immature neurons. MAP2 consists of several isoforms, including two high molecular weight forms, termed MAP2 a and b, and a lower molecular weight form termed MAP2c (Garner et al., 1988). In most neurons, MAP2 a and b are restricted to the somatodendritic compartment, whereas MAP2c is present in both the somatodendritic and axonal compartments (Meichsner et al., 1993). Immunoblotting of whole-cell SDS extracts of 1-d-old cultures of rat sympathetic neurons with a mouse monoclonal $\mathrm{Ab}$ that recognizes MAP2 a, b, and c reveals all three forms of MAP2, with MAP2c accounting for $\sim 35 \%$ of the total MAP2 immunoreactivity (by comparison, MAP2c in high-speed supernates of P7 rat brain accounts for $\sim 67 \%$ of the total MAP2 immunoreactivity) (data not shown). Abs that recognize MAP2 a and b, but not MAP2c, localize principally to the cell body; faint staining is seen in the axon, and this is mostly in its proximal part (data not shown) (see also Peng et al., 1986; Black et al., 1996), and Abs that recognize MAP2c as well as MAP2 a and b give a similar pattern (data not shown). Staining of Ab-injected cells with either MAP2 Abs produced images indistinguishable from those obtained from control cells (data not shown), indicating that the immunodepletion of tau did not appreciably affect MAP2 distribution. Similarly, injection of tau Abs had no detectable effect on the staining pattern for MAP1b (data not shown).

Finally, several observations indicate that tau can interact with actin filaments and that tau can mediate interactions between actin filaments and MTs (for review, see Delacourte and Buee, 1997). Also, DiTella et al. (1994) have reported that the depletion of tau in cultured cerebellar neurons by using antisense strategies alters actin filament organization in growth cones. As an initial approach to evaluate whether the immunodepletion of tau alters interactions between MTs and actin filaments in growth cones of the neurons used in the present studies, we have compared the arrangement of these polymer systems in the growth cones of Ab-injected and control neurons. Actin filaments and MTs have a fairly characteristic arrangement in growth cones in which actin filaments are concentrated in the peripheral parts of growth cones, whereas MTs are most abundant in the central domain of growth cones, with their distal ends overlapping the proximal regions of the actin filament-rich regions. Inactivation of tau by $\mathrm{Ab}$ injection had no obvious effect on the arrangements of these polymer systems, at least as viewed by immunofluorescence microscopy (data not shown). The reason for the difference between our results and those of DiTella et al. (1994) is not known. In this regard, the culture conditions used in the present studies are very different from those used by DiTella et al. (1994), and culture conditions can influence the response of neurons to tau depletion caused by treatment with antisense (DiTella et al., 1996).

\section{Axon growth parameters in control and Ab-injected cells}

On the basis of the images presented in Figures 1 and 2, it is apparent that Ab-injected cells extend axons and that the morphology of the cells in terms of axon length, number of axons per cell, and number of branches per axon resembles that of control cells. Quantitative analyses confirm this impression (see Tables 2-4). Initial analyses suggested that axons of cells injected with tau Abs grew slightly faster than the axons of uninjected cells (Table 2). This difference apparently was attributable to some aspect of the injection procedure and was not dependent on the injection of tau Abs. This was revealed by comparing uninjected cells with cells injected with either nonimmune or tau Abs. In this case the axon growth rates of cells injected with either $\mathrm{Ab}$ were indistinguishable from each other but were slightly faster than the rates of uninjected cells. The basis for this difference is unknown. 
Tyrosinated Tubulin

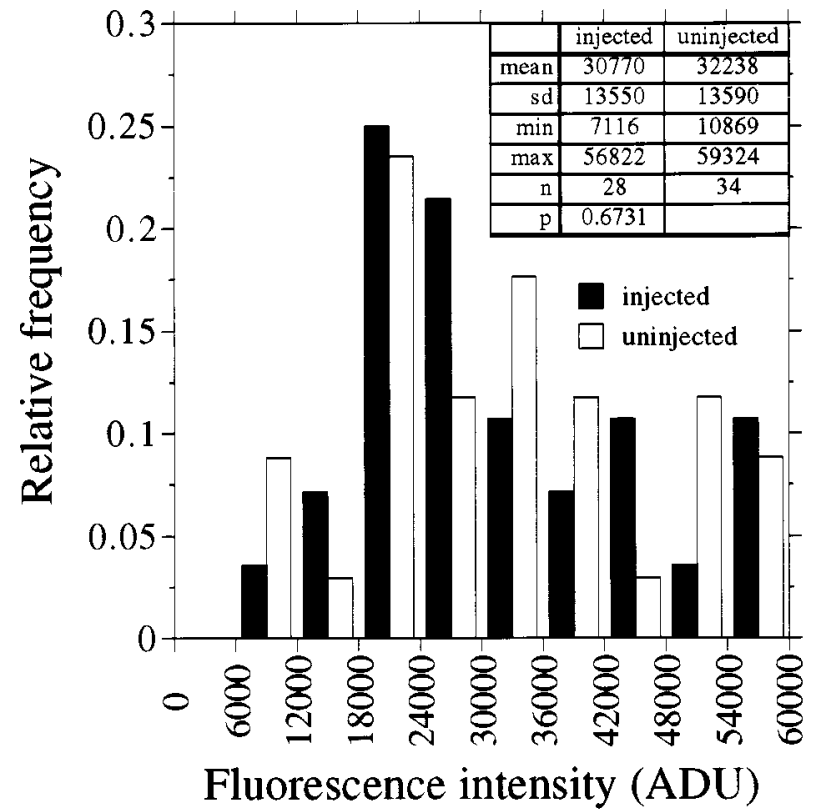

Acetylated Tubulin

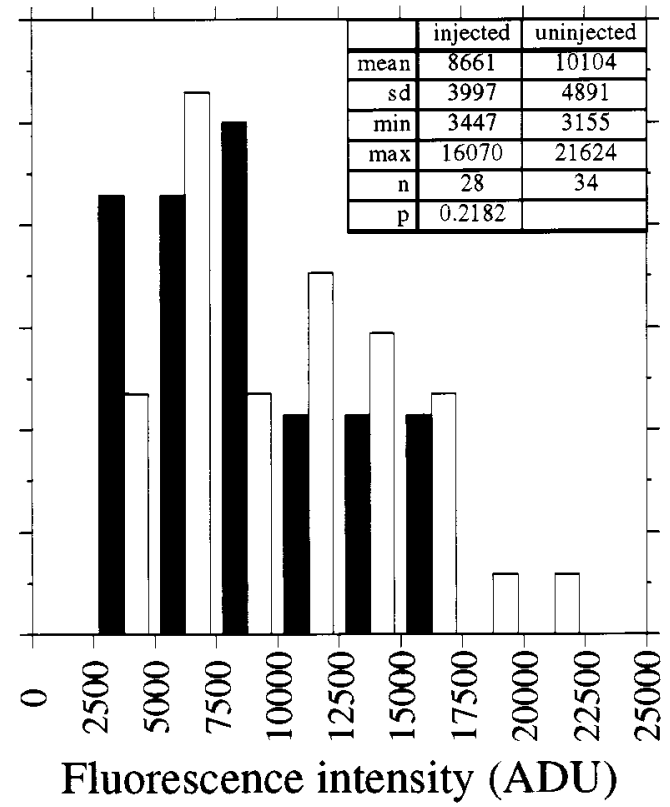

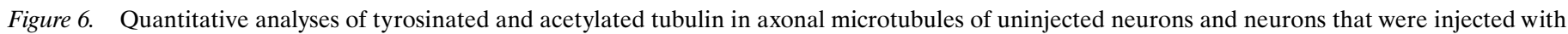

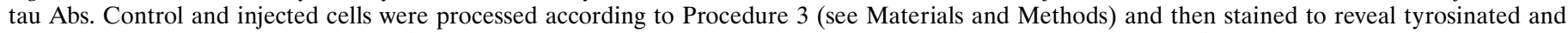

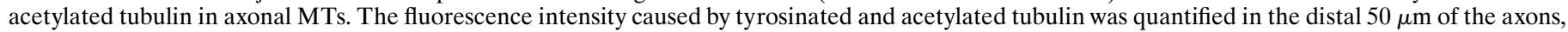

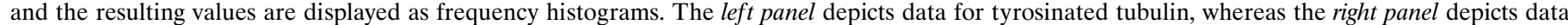

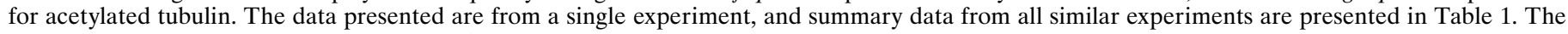

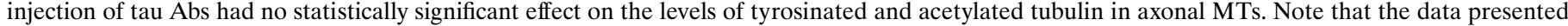
in this figure are from an entirely separate experiment from the data presented in Figure 5.

Given the slightly faster growth rate of injected cells, it is reasonable to expect that injected cells should produce slightly more axons than uninjected cells, and this was borne out in three of four experiments (Table 3). Additional experiments in which nonimmune Abs were injected indicated that this difference was related to some aspect of the injection procedure and was not dependent on the specific injection of tau Abs. Finally, the number of axons produced per cell and the extent of axon branching by cells injected with tau Abs were very similar to those of uninjected cells (Table 4).

In evaluations of the significance of these data, it is relevant to consider the time course of tau sequestration after the injection of tau Abs relative to the time course of axon growth. We have examined cells 1-2 hr after stimulating injected cells to initiate axon growth. Tau aggregates are readily apparent in cells at this time, whether or not they have initiated axon growth. In cells that have initiated axon growth, tau staining in the nascent axons is reduced substantially as compared with controls. Thus, tau sequestration is well developed throughout the period that injected cells initiate and then elongate their axons. Taken together, all of these data indicate that the immunodepletion of tau does not affect axonal growth or morphology detectably, at least under the conditions of the present experiments. It should be noted, however, that, because of the considerable variation in growth behavior among the neurons, it is possible that differences exist between control and Ab-injected cells but that they were masked by the cell-to-cell variation. Such differences, if they exist, must be relatively subtle, because the means as well as the overall distribution of values for control and Ab-injected cells are so similar to each other. In this regard, the limited effect of tau inactivation on axon growth that was observed in the present studies confirms recent antisense experiments that showed that tau is not required for axon growth by cultured cerebellar neurons (DiTella et al., 1996).

Finally, in a small number of cases we examined axon growth over a more prolonged period. In these analyses the Ab-injected cells were maintained for $\sim 20 \mathrm{hr}$ after axon growth was stimulated, and then they were fixed and stained to reveal MTs and tau. Tau staining in injected cells was confined mainly to aggregates in the cell body, with little if any tau staining in the axons (data not shown). Thus, tau sequestration continues for at least $20 \mathrm{hr}$ after $\mathrm{Ab}$ injection. Morphologically, control cells as well as Ab-injected cells have long, highly branched axons that are much longer than those seen at 4-6 hr after injection. The extent of axon growth over this period was so great that the axons formed a complex network that covered much of the surface of the coverslip. Because individual axons frequently crossed over and fasciculated with other axons, it was impossible to measure reliably the extent of axon production by control or injected neurons. Nonetheless, visual inspection of the injected cells indicates that axon growth proceeds vigorously for at least $20 \mathrm{hr}$ after the immunodepletion of tau.

\section{DISCUSSION}

We have microinjected purified Abs against tau into neurons before they initiated axon growth. The injected Abs sequestered tau and prevented it from binding to MTs. Despite this, the injected neurons extended axons that resembled those of control cells, and the axons contained normal-appearing MT arrays with apparently normal dynamic behavior. Given that tau has relatively potent effects on MT assembly and stability in vitro (Trinczek et al., 1995), these in vivo results are unexpected. In consid- 


\section{Injected}

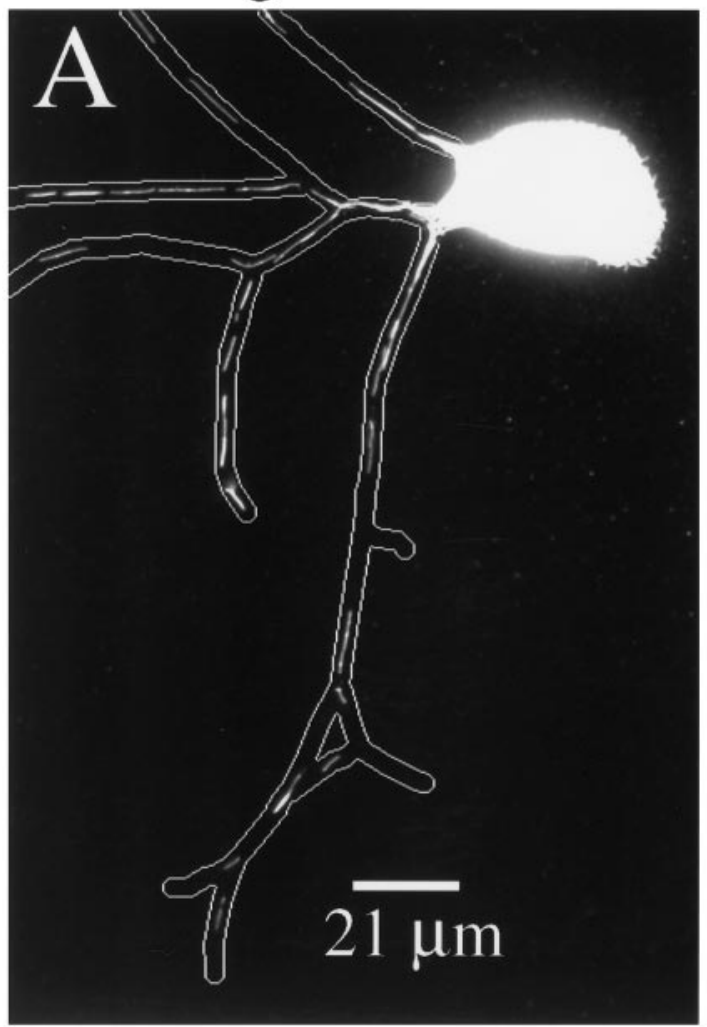

\section{Uninjected}

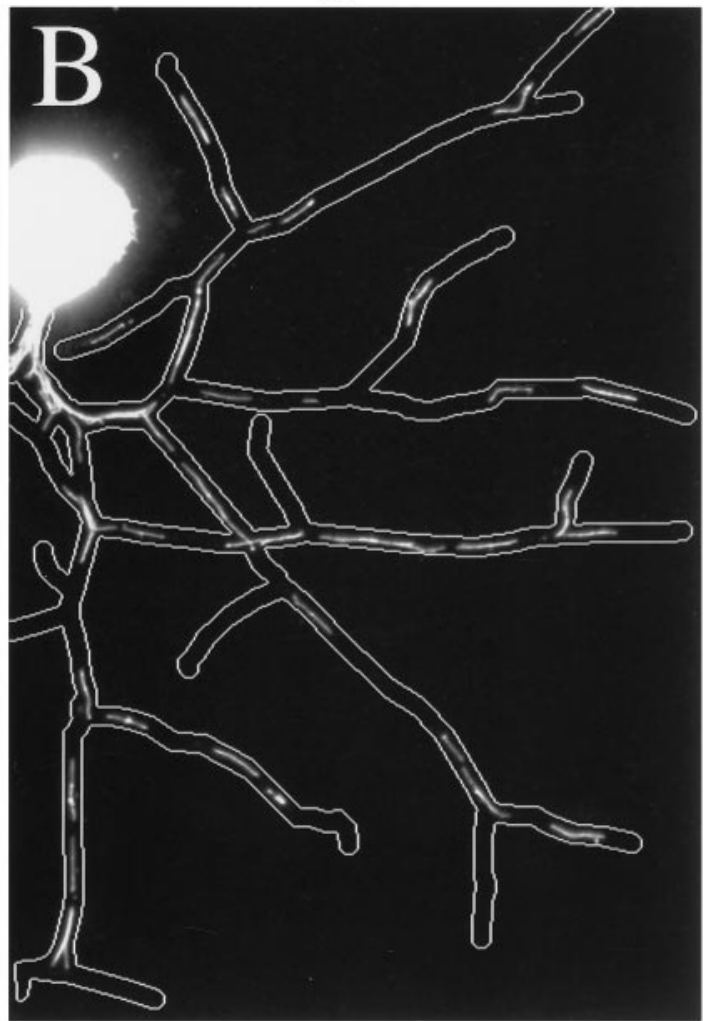

Figure 7. MT regrowth in uninjected and anti-tau-injected cells. Control and anti-tau-injected cells were stimulated to extend axons, treated with 5 $\mu \mathrm{g} / \mathrm{ml}$ Nocodazole for $\sim 30 \mathrm{~min}$, injected with Bt-tub, and then incubated a further $90-120 \mathrm{~min}$. MT regrowth was initiated by rinsing the cultures free of Nocodazole. At 10-15 min later the cells were extracted, fixed, and then stained to reveal Bt-tub in MTs, actin filaments, and injected Abs. The $\mathrm{Ab}$-injected cell had a large aggregate of injected $\mathrm{Ab}$ in the cell body (data not shown), indicating the aggregation of tau in the cell body (for details, see the legend to Table 1). The actin staining was used to create an outline of the axon, which is shown superimposed on the Bt-tub staining images. $A$ shows Bt-tub in MTs of a control cell, whereas $B$ shows Bt-tub in MTs of a cell that has been injected with tau Abs. Contrast and brightness were optimized to reveal axonal staining for Bt-tub; as a result, somal staining is saturated. Note the presence of segments of assembled Bt-tub scattered along the axons, interspersed with regions containing no Bt-tub.

\section{Table 2. Effects of tau Ab injection on axon growth rate}

\begin{tabular}{lll}
$\begin{array}{l}\text { Experiment } \\
\text { number }\end{array}$ & $\begin{array}{l}\text { Average growth rate } \\
(\mu \mathrm{m} / \mathrm{hr}) \text { mean } \pm \\
\mathrm{SD}\left(n^{*}\right)\end{array}$ \\
\hline 1 & Uninjected & $43 \pm 23(27)$ \\
& Anti-tau injected & $52 \pm 29(23)$ \\
Uninjected & $43 \pm 17(26)$ \\
& Injected & \\
& Nonimmune & $48 \pm 20(32)$ \\
& Anti-tau & $48 \pm 23(24)$
\end{tabular}

$\overline{\text { Axon growth rates were determined as described in Materials and Methods. }{ }^{*} n \text {, The }}$ total number of axons analyzed. Growth rates of cells injected with tau Abs were not statistically different from those of cells injected with nonimmune $\mathrm{Ab}(p>0.1)$.

ering their significance, we believe that it is important to evaluate the extent to which $\mathrm{Ab}$ injection prevents tau from binding to MTs. Using two different antibodies and fixation and staining conditions that were optimized to reveal tau in cultured sympathetic neurons (Black et al., 1996), we reduced tau staining in the axons of neurons injected with tau Abs by $\sim 90 \%$ as compared with controls (see Fig. 3). However, we consider this a minimal estimate, and we believe that the true extent of tau immunodeple-
Table 3. Effects of tau Ab injection on the total length of axon produced

\begin{tabular}{lll}
$\begin{array}{l}\text { Experiment } \\
\text { number }\end{array}$ & $\begin{array}{l}\text { Total axon length } \\
(\mu \mathrm{m}) \text { mean }+ \text { SD }\left(n^{*}\right)\end{array}$ \\
\hline 1 & Uninjected & $1114 \pm 436(23)$ \\
2 & Anti-tau injected & $1421 \pm 744(24)$ \\
& Uninjected & $1436 \pm 429(18)$ \\
3 & Anti-tau injected & $1488 \pm 565(15)$ \\
& Uninjected & $1572 \pm 780(43)$ \\
4 & Anti-tau injected & $1940 \pm 764(26)$ \\
& Uninjected & $2158 \pm 590(12)$ \\
& Injected & \\
& Nonimmune & $2394 \pm 635(12)$ \\
& Anti-tau & $2580 \pm 839(11)$
\end{tabular}

The total length of all axons and their branches was determined for injected and uninjected cells. ${ }^{*} n=$ the total number of axons analyzed. The total length of axon produced by cells injected with tau Abs did not differ significantly from that generated by cells injected with non-immune $\mathrm{Ab}$ (see Results for additional details).

tion is $>90 \%$. The quantitative analyses did not take into account the nonspecific staining that occurred with our procedures. The non-neuronal cells present in the cultures provide an indication of this staining, because they do not express tau. A finite glow is 


\begin{tabular}{|c|c|c|c|}
\hline $\begin{array}{l}\text { Experiment } \\
\text { number }\end{array}$ & & $\begin{array}{l}\text { Number of axons/ } \\
\text { neuron means }+ \\
\operatorname{SD}\left(n^{*}\right)\end{array}$ & $\begin{array}{l}\text { Number of } \\
\text { branches/axon } \\
\text { means }+ \text { SD }\end{array}$ \\
\hline \multirow[t]{2}{*}{1} & Uninjected & $3 \pm 2(21)$ & $5 \pm 6$ \\
\hline & Anti-tau injected & $4 \pm 2(23)$ & $6 \pm 6$ \\
\hline \multirow[t]{2}{*}{2} & Uninjected & $3 \pm 2(25)$ & $8 \pm 9$ \\
\hline & Anti-tau injected & $2 \pm 1(21)$ & $9 \pm 8$ \\
\hline \multirow[t]{2}{*}{3} & Uninjected & $3 \pm 1(20)$ & $2 \pm 2$ \\
\hline & Anti-tau injected & $4 \pm 2(17)$ & $3 \pm 3$ \\
\hline \multirow[t]{4}{*}{4} & Uninjected & $4 \pm 2(12)$ & $9 \pm 10$ \\
\hline & Injected & & \\
\hline & Nonimmune & $4 \pm 2(12)$ & $12 \pm 11$ \\
\hline & Anti-tau & $3 \pm 2(11)$ & $14 \pm 14$ \\
\hline
\end{tabular}

We counted the total number of axons extending from the cell body and the total number of branches per axon for both injected and uninjected cells. * $n$, The total number of axons analyzed. Ab injection had no statistically significant effect on the numbers of axons per neuron nor on the number of branches per axon.

associated with these cells (see Fig. 2), and this closely resembles that associated with axons of Ab-injected cells. Thus, a substantial portion of the fluorescence that is associated with injected axons is nonspecific.

Nonetheless, some tau may be present in the axons of injected cells. However, it is unlikely that this tau is of functional concern. First, relatively large quantities of tau Abs were injected into the cells (see Materials and Methods), and these Abs became distributed throughout the axon. Thus, any tau present in the axon would be coated with the tau Abs. Typically, we injected a mix of two tau Abs for which the epitopes span approximately one-third of the entire protein. Thus, even if tau is present in the axon, such extensive coating with Abs is likely to impair tau function(s), if not to render it entirely nonfunctional because of local immunoprecipitation and/or the blockage of functionally important sites. Second, tau decorates MTs in growth cones of control cells, and this decoration is detected readily by immunofluorescence procedures (see Fig. 2). In growth cones of injected cells stained for tau, fluorescence does not localize to MTs (see Fig. 2). Thus, the amount of tau in these growth cones that is capable of binding to MTs is too small to stain MTs above background.

Collectively, these considerations indicate that little if any functional tau is present in the axons of Ab-injected cells. Nonetheless, it is relevant to ask whether this amount of tau is sufficient to maintain its normal function(s). Although a definitive answer to this question is not possible, we can estimate that residual tau present in the axons of injected cells represents at most $10 \%$ and probably a much smaller percentage of that normally present. For this amount of tau to maintain the normal function(s) of tau means that the neuron typically produces at least a 10 -fold excess of tau, and more probably a 50- to 100 -fold excess. For a neuron to produce such an excess of tau normally seems implausible (see also Wang et al., 1996).

Tau has potent effects on MT assembly and stability in the test tube (Trinczek et al., 1995). Tau is also present in developing neurons, where it associates with MTs. Thus, it is reasonable to expect that tau will promote MT assembly and stability in growing neurons. However, we detected no such effects after acute immunodepletion of tau in cultured sympathetic neurons. One possible explanation for this lack of effect is that these neurons contain other MAPs that have a sufficient redundancy of function with respect to tau so that they can compensate effectively for the absence of tau. In this regard, DiTella et al. (1996) have suggested that tau and MAP1b are functionally redundant in growing cerebellar neurons. They showed that the suppression of both MAP1b and tau by using antisense strategies prevented axon growth by cultured cerebellar neurons, whereas suppression of just one MAP did not block axon growth, although growth was diminished as compared with controls. In these studies the functions of tau and MAP1b were evaluated in terms of axon growth; the data indicate that tau and MAP1b both participate in axon growth but that one of these is sufficient to sustain growth (DiTella et al., 1996). However, the specific functions of tau and MAP1b were not examined directly, and so the extent to which specific cellular actions of these proteins are redundant is not known.

In the context of the present studies the hypothesis of functional redundancy implies that tau and at least one other MAP have similar effects on MT assembly and stability so that the loss of tau is compensated for by the remaining MAP(s). Cultured sympathetic neurons contain a variety of MAPs in addition to tau, some of which are present in the axon and growth cone together with tau (Peng et al., 1986; Black et al., 1994). Thus, it is possible that one or more MAPs may compensate for the removal of tau with regard to MT assembly and stability; hence, we observed no effect of tau immunodepletion on these parameters. Implicit in this view is that tau normally contributes to the dynamic behavior of MTs in growing axons. However, several observations suggest an alternative view in which tau is not a major determinant of MT stability in growing axons. Most compelling in this regard is that the most dynamic polymer of growing axons, which is located distally in the axon shaft and in the growth cone (Lim et al., 1989; Brown et al., 1992; Baas et al., 1993; Edson et al., 1993; Kempf et al., 1996; Li and Black, 1996), is also the most enriched in tau (Black et al., 1996). These observations are exactly opposite of those expected on the basis of the hypothesis that the stability of axonal MTs is a direct function of their content of tau.

Other observations also question whether tau in growing axons functions principally to promote MT assembly and stability. For example, tau consists of a variety of isoforms that differ substantially in their binding affinity for MTs and in their ability to promote MT assembly and stability (Lovestone and Reynolds, 1997). The isoform composition of tau is regulated developmentally, and, in test tubes the specific isoforms in immature neurons have lower MT binding affinities and assembly-promoting and stabilizing activities than those in mature neurons (Lovestone and Reynolds, 1997). Thus, mechanisms operate in developing neurons to actively reduce the affinity of tau for MTs and thereby diminish its ability to promote MT assembly and stability. Presumably, these mechanisms account for the observation that the interaction of tau with MTs in growing axons is labile under a variety of conditions that preserve MTs and the interactions of other MAPs with MTs (Black et al., 1996; Kempf et al., 1996).

In the test tube the assembly-promoting and stabilizing activity of tau increases with the increasing molar ratio of tau to tubulin (Cleveland et al., 1977; Bre and Karsenti, 1990; Panda et al., 1995). If tau promotes MT assembly and stability in growing axons, then varying the tau-to-tubulin ratio should have concomitant effects on the proportion of tubulin in polymer. Cultured sympathetic neurons provide a natural test of this prediction, because the ratios of total tau to total tubulin and assembled tau to assembled tubulin increase progressively along the axon, 
reaching a peak distally that is greater than that found proximally (Black et al., 1996). Despite these regional differences in the tau-to-tubulin ratio, the distribution of tubulin between protomer and polymer is similar in proximal and distal axonal regions. Thus, contrary to the prediction, the extent of tubulin assembly along the axon is not related directly to the tau-to-tubulin ratio. Similarly, overexpressing tau in non-neuronal cells (Barlow et al., 1994) or PC12 cells grown without nerve growth factor (EsmaeliAzad et al., 1994) does not increase the proportion of tubulin in polymer. Finally, in cells expressing green fluorescent protein tau, MTs exhibit dynamic behavior although they are decorated all along their length with tau (Kaech et al., 1996).

These several observations suggest that tau is not a principal determinant of MT assembly or stability in growing axons, and the present results provide experimental support for this view by showing that the immunodepletion of tau has no measurable effect on several properties of MTs in growing axons that reflect on their dynamic behavior. These data do not preclude the possibility that tau influences MT dynamics in growing axons. Rather, they indicate that the effects of tau in this regard are quantitatively modest. The situation in adult axons may be quite different because, as indicated above, the specific tau isoforms present in adult neurons are, in general, more potent than those in developing neurons with regard to their assembly-promoting and stabilizing activities.

The suppression of the assembly-promoting and stabilizing activities of tau in developing neurons may facilitate the proper establishment of synaptic connections. Specifically, the dynamic behavior of MTs in growing axons and their growth cones strongly influences growth cone motility and pathfinding behaviors such as growth cone turning (Tanaka et al., 1995; Rochlin et al., 1996; Williamson et al., 1996; Challacombe et al., 1997; Gallo, 1998). Thus, suppressing the MT assembly and stabilizing activities of tau may be one strategy used by growing neurons to foster the behaviors that are necessary for elongating axons to find their synaptic partners (see also Goode and Feinstein, 1994).

Given that the MT assembly and stabilizing activities of tau are actively suppressed in developing neurons, it is reasonable to infer that tau is expressed to perform other functions. These functions presumably involve MTs in the axon and growth cone, because tau binds MTs in these regions. In this regard, a variety of MT-related activities of tau has been discussed in addition to its effects on MT assembly and stability. For example, under in vitro conditions, tau affects mechanical properties of MTs (Felgner et al., 1997) and mediates interactions between MTs and other cytoskeletal polymers (for review, see Maccioni et al., 1995), whereas the overexpression of tau in various cell types has provided evidence for tau bundling MTs (Kanai et al., 1992) and interacting with the plasma membrane, possibly linking MTs to the membrane (Brandt et al., 1995). Which, if any, of these activities is relevant to tau function in growing axons is unknown. Regardless of the specific function of tau, the observation that tau is not required for axon growth (Harada et al., 1994; DiTella et al., 1996; this study) indicates that tau does not perform unique functions that are essential to neuronal morphogenesis. Either tau is involved in functions that are not essential for this or tau cooperates with other partners to perform essential function(s), and these partners are able to compensate effectively for the absence of tau.

\section{REFERENCES}

Baas PW, Black MM (1990) Individual microtubules in the axon consist of domains that differ in both composition and stability. J Cell Biol 111:495-509.

Baas PW, Brown A (1997) Slow axonal transport: the polymer transport model. Trends Cell Biol 7:380-384.

Baas PW, Ahmad FJ, Pienkowski TP, Brown A, Black MM (1993) Sites of stabilization for axonal microtubules. J Neurosci 13:2177-2185.

Barlow S, Gonzalez-Garay ML, West RR, Olmsted JB, Cabral F (1994) Stable expression of heterologous microtubule-associated proteins (MAPs) in Chinese hamster ovary cells: evidence for differing roles of MAPs in microtubule organization. J Cell Biol 126:1017-1029.

Black MM (1987) Comparison of MAP-2 and tau on the packing density of assembled microtubules. Proc Natl Acad Sci USA 84:7783-7787.

Black MM (1994) Microtubule assembly and transport cooperate to generate the microtubule array of the axon. Prog Brain Res 102:61-77.

Black MM, Kurdyla JT (1983) Microtubule-associated proteins of neurons. J Cell Biol 97:1020-1028.

Black MM, Slaughter T, Fischer I (1994) Microtubule-associated protein $1 \mathrm{~b}$ (MAP1b) is concentrated in the distal region of growing axons. J Neurosci 14:857-870.

Black MM, Slaughter T, Moshiach S, Obrocka M, Fischer I (1996) Tau is enriched on dynamic microtubules in the distal region of growing axons. J Neurosci 16:3601-3619.

Brandt R, Leger J, Lee G (1995) Interaction of tau with the neural plasma membrane mediated by tau's amino-terminal projection domain. J Cell Biol 131:1327-1340.

Bre MH, Karsenti E (1990) Effects of brain microtubule-associated proteins on microtubule dynamics and the nucleating activity of centrosomes. Cell Motil Cytoskeleton 15:88-98.

Brown A, Slaughter T, Black MM (1992) Newly assembled microtubules are concentrated in the proximal and distal regions of growing axons. J Cell Biol 119:867-882.

Brown A, Li Y, Slaughter T, Black MM (1993) Composite microtubules of the axon: quantitative analysis of tyrosinated and acetylated $\alpha$-tubulin along axonal microtubules. J Cell Sci 104:339-352.

Challacombe JF, Snow DM, Letourneau PC (1997) Dynamic microtubule ends are required for growth cone turning to avoid an inhibitory guidance cue. J Neurosci 17:3085-3095.

Cleveland DW, Hwo S-Y, Kirschner MW (1977) Purification of tau, a microtubule-associated protein that induces assembly of microtubules from purified tubulin. J Mol Biol 116:207-225.

Delacourte A, Buee L (1997) Normal and pathological tau proteins as factors for microtubule assembly. Int Rev Cytol 171:167-224.

DiTella M, Feiguin F, Morfini G, Caceres A (1994) Microfilamentassociated growth cone component depends upon tau for its intracellular localization. Cell Motil Cytoskeleton 29:117-130.

DiTella MC, Feiguin F, Carri N, Kosik KS, Caceres A (1996) MAP1b/ tau functional redundancy during laminin-enhanced axonal growth. J Cell Sci 109:467-477.

Drubin DG, Feinstein SC, Shooter EM, Kirschner MW (1985) Nerve growth factor-induced outgrowth in PC12 cells involves the coordinate induction of microtubule assembly and assembly promoting factors. J Cell Biol 101:1799-1807.

Edson KJ, Lim S-S, Borisy GG, Letourneau PC (1993) FRAP analysis of the stability of the microtubule population along the neurites of chick sensory neurons. Cell Motil Cytoskeleton 25:59-72.

Esmaeli-Azad B, McCarty JH, Feinstein SC (1994) Sense and anti-sense transfection analysis of tau function: tau influences net microtubule assembly, neurite outgrowth, and neuritic stability. J Cell Sci 107:869-879.

Felgner H, Frank R, Biernat J, Mandelkow EM, Mandelkow E, Ludin B, Matus A, Schliwa M (1997) Domains of neuronal microtubuleassociated proteins and flexural rigidity of microtubules. J Cell Biol 138:1067-1075.

Gallo G (1998) Involvement of microtubules in the regulation of neuronal growth cone morphologic remodeling. J Neurobiol 35:121-140.

Garner CC, Brugg B, Matus A (1988) A 70-kilodalton microtubuleassociated protein (MAP2c), related to MAP2. J Neurochem 50:609-615.

Goode BL, Feinstein SC (1994) Identification of a novel microtubule binding and assembly domain in the developmentally regulated interrepeat region of tau. J Cell Biol 124:769-782. 
Harada H, Oguuchi K, Okabe S, Kuno J, Terada S, Oshima T, SatoYoshitake R, Takel Y, Noda T, Hirokawa N (1994) Altered microtubule organization in small-caliber axons of mice lacking tau protein. Nature 369:488-491.

Kaech S, Ludin B, Matus A (1996) Cytoskeletal plasticity in cells expressing neuronal microtubule-associated proteins. Neuron 17:1189-1199.

Kanai Y, Chen J, Hirokawa N (1992) Microtubule bundling by tau proteins in vivo: analysis of functional domains. EMBO J 11:3953-3961.

Kempf M, Clement A, Faissner A, Lee G, Brandt R (1996) Tau binds to the distal axon early in development of polarity in a microtubule and microfilament-dependent manner. J Neurosci 16:5583-5592.

Lee G, Rook SL (1992) Expression of tau protein in nonneuronal cells: microtubule binding and stabilization. J Cell Sci 102:227-237.

Li Y, Black MM (1996) Microtubule assembly and turnover in growing axons. J Neurosci 16:531-544.

Lim S-S, Sammak PJ, Borisy GG (1989) Progressive and spatially differentiated stability of microtubules in developing neuronal cells. J Cell Biol 109:253-263.

Lovestone S, Reynolds CH (1997) The phosphorylation of tau; a critical stage in neurodevelopment and neurodegenerative processes. Neuroscience 78:309-324.

Maccioni RB, Tapia L, Cambiazo V (1995) Functional organization of tau proteins during neuronal differentiation and development. Braz J Med Biol Res 28:827-841.

Meichsner M, Doll T, Reddy D, Weisshaar B, Matus A (1993) The low molecular weight form of microtubule-associated protein 2 is transported into both axons and dendrites. Neuroscience 54:873-880.

Panda D, Goode BL, Feinstein SC, Wilson L (1995) Kinetic stabilization of microtubule dynamics at steady state by tau and microtubule-binding domains of tau. Biochemistry 34:11117-11127.

Papasozomenos SC, Binder LI (1987) Phosphorylation determines two distinct species of tau in the central nervous system. Cell Motil $\mathrm{Cy}$ toskeleton 8:210-226.

Peng I, Binder LI, Black MM (1985) Cultured neurons contain a variety of microtubule-associated proteins. Brain Res 361:200-211.

Peng I, Binder LI, Black MM (1986) Biochemical and immunochemical analyses of cytoskeletal domains of neurons. J Cell Biol 102:252-262.

Rochlin MW, Wickline KM, Bridgman PC (1996) Microtubule stability decreases axon elongation but not axoplasm production. J Neurosci 16:3236-3246.

Schoenfeld TA, Obar RA (1994) Diverse distribution and function of fibrous microtubule-associated proteins in the nervous system. Int Rev Cytol 151:67-137.

Slaughter T, Wang J, Black MM (1997) Microtubule transport from the cell body into the axons of growing neurons. J Neurosci 17:5807-5819.

Tanaka E, Ho T, Kirschner MW (1995) The role of microtubule dynamics in growth cone motility and axonal growth. J Cell Biol 128:139-155.

Trinczek B, Biernat J, Baumann K, Mandelkow E-M, Mandelkow E (1995) Domains of tau protein, differential phosphorylation, and dynamic instability of microtubules. Mol Biol Cell 6:1887-1902.

Wang XM, Peloquin JG, Zhai Y, Bulinski JC, Borisy GG (1996) Removal of MAP4 from microtubules in vivo produces no observable phenotype at the cellular level. J Cell Biol 132:345-357.

Williamson T, Gordon-Weeks PR, Schachner M, Taylor J (1996) Microtubule reorganization is obligatory for growth cone turning. Proc Natl Acad Sci USA 93:15221-15226. 